\title{
The Loss from Imperfect Value Functions in Expectation-Based and Minimax-Based Tasks
}

\author{
MATTHAS HEGYR \\ heger@informatik.unï-brerren.de

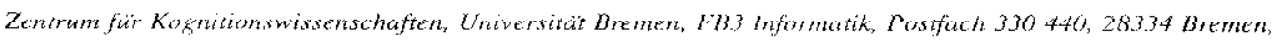 \\ Germany:
}

Editor: Leslic Pack Kaelbling

\begin{abstract}
Many reinforcement learning (RL.) algorithms approximate an optimal value funcrion. Once the function is known, it is easy to determine an optimal policy. For most real-world applications, however, the value function 15 too complex to be represented by lookup tables, making it necessary to use function approximators such as neural networks. In this casc, convergence to the optimal value function is no longer guaranteed and it becomes important to know to which extent performance diminishes when one uses approximate value functions insread of optimal ones. This problem has recently been discussed in the context of expectation-based Markov decision problems. Our analysis generalizes this work to mimimax-based Markov decision problems, yields new results for expectation-based tasks, and shows how minimax-based and expectation-basted Markov decision problems relate.
\end{abstract}

Keywords: Reinforement Learning, Dynamic Programming. Performance Bounds, Minirnax Algorithms, Q-Learning

\section{Introduction}

Reinforcement learning (RL) is learning to solve decision problems from experience: an agent interacts with its environment (world) and receives reinforcement signals as punishments for its actions. Its task is to find a behavior that minimizes the punishment, specified as rules that tell the agent which action to choose in every possible situation. (The reinforcement signals can also be interpreted as rewards. This is equivalent to their interpretation as punishments, except that the agent now has to maximize the reward.)

'The interaction of the agent with its environment is often modeled as a special kind of stochastic process, namely a Markov decision process (MDP). $S$ is the set of states of the environment as the agent perceives it. $A$ the set of actions that the agent has available, and $C$ the set of scalar reinforcement signals, which we call immediate costs because they can be interpreted as the effort required to execute the actions. In this paper, we limit ourselves to finite sets $S$ and $A$ that are subsets of $N$. We assume that the elements of $C<R$ are countable, bounded, and nonnegative. Furthemore, the agent does not have all actions available in every state. The nonempty sets $A(i)=A$ denote the set of admissible actions in states $i$

The interaction of the agent with its environment takes place in episodes, where episodes correspond to time steps: First, the agent observes the starting state i $S$. It then has to execute an action a $5 A(j)$, which causes a state transition from state i 10 a successor state $j \in S$. Finally, the agent receives the reinforiement signal $r \in C$. 
MDPs make the important assumption that the probability distribution over the successor states depends only on the starting state $i$ and the exccuted action a but not on $t$ or any previous episodes. $F_{S}(i, a, j)$ denotes the probability that the successor state of an cpisode is $j$ if action $a$ is executed in starting state $s$. Similarly, MDPs assume that the probability that the immediate cost of episode $t$ cquals a given number $r \in C$ depends only on the starting state, the executed action, and the successor state of that episode, but neither on $t$ nor past episodes. $P_{C}(i, a, j, r)$ denotes the probability that the immediate cost of an episode is $r$ for a given starting state $i$, action $a$, and successor state $j$. Furthermore, MDPs assume that the starting state of an episode is identical to the successor state of the previous episode. If the MDP is deterministic, then the successor state $j_{i}^{a}$ and the immediate cost $c_{i}^{a}$ are uniquely determined by the starting state $i$ and the executed action $a$.

The behavior of the agent is specified by a policy, which is a mapping from situations to actions. In general, the action could depend on the current time, the current state of the agent, and all previous states and actions. Furthermore, the action could be selected probabilistically. Stationary policies determine which action to execute based only on the current state of the agent, but prove to be very powerful.

The slates and immediate costs are random variables because of the probabilistic nature of the immediate costs and state transitions. These random variables essentially depend on the policy that the agent follows. Therefore, we use the following notation: $I_{t}^{\text {r }}$ denotes the starting state and $C_{t}^{\pi}$ the immediate cost, where $\pi$ and $t$ describe the policy of the agent and the time index of the episode, respectively.

There are two problems that make it difficult to define what an optimal policy is. The first problem is often called delayed reinforcement, because actions may reveal their consequences only many time steps after they have been executed. Hence, the action that has the lowest immediate cost is not neccssarily best from a global point of view. Therefore, one should consider all immediate costs, including the future costs, which is usually done by evaluating policies according to their return

$$
R_{\gamma}^{\pi}=\sum_{\tau=0}^{\infty} \gamma^{\tau} C_{\gamma}^{\pi}
$$

where $0 \leq y<1$ is a discount factor that keeps the sum finite and has the effect that costs are weighted less if they are obtained farther into the future.

In deterministic domains, the return of a policy can already be used to measure its performance. In this case, a policy is optimal if it minimizes the return. Unfortunately, there is a second problem, since the return is usually not a real number, but a random variable. In probabilistic domains, using the same policy repeatedly in the same starting state can result in different returns. The common way to handle this problem is to use the expected value of the return as performance measure (Barto, Sutton \& Anderson, 1983; Watkins, 1989). According to this so-called expected value criterion, a policy is optimal if it minimizes the expected value of the return. In operations research and decision theory, however, it is well known that it is not always reliable and can even be very misleading to use the expected value as decision criterion (e.g., Taha, 1987). In (Heger, 1994a), Heger presented dynamic programming algorithms and a RI algorithm callod 


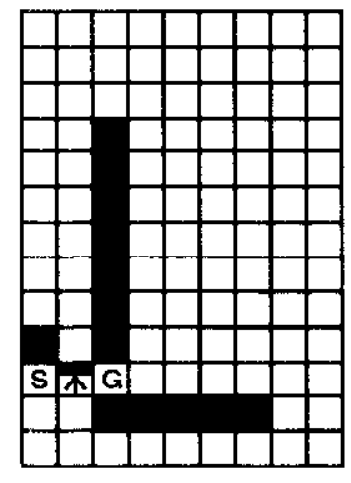

Figure 1a

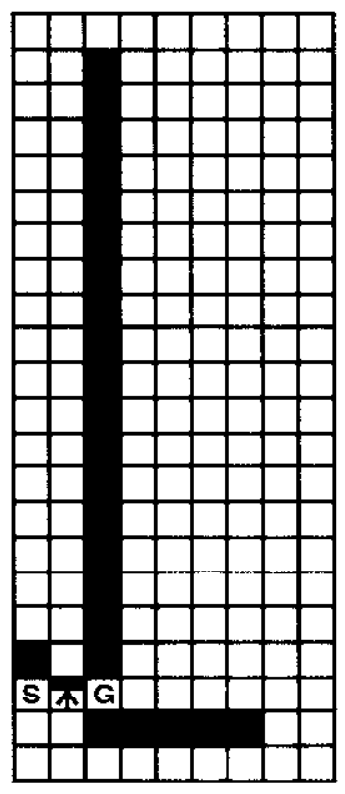

Figure 1b

Figure 1. Two Grid Worlds where Expectation-Optimal and Minimax Optimal Policies Differ

Q-hat-learning that are based on the minimax criterion. According to this criterion, a policy is optimal if it minimizes the worst-case return. The main contribution of this paper is to present new results for minimax-based tasks. The following two examples are intended to convince the reader that it is worthwhile to consider the minimax criterion as an alternative to the expected-value criterion.

In the stochastic domain of Figure 1a, the minimax optimal policy differs from the expectation-optimal one. An agent (robot) has to navigate on the grid from location $\mathrm{S}$ (start) to $G$ (goal). Dark squares represent obstacles; so does the perimeter of the grid. In each square, the agent has the four actions UP, DOWN, LEFT, and RIGHT available that take it to the corresponding adjacent square, unless the square is occupied by an obstacle, in which case the agent does not change location. Every state transition has an immediate cost of one except for state transitions from the goal state into itself, which have cost zero. The square with the arrow is a probabilistic one-way door. It can be passed through only in the direction of the arrow and behaves like an obstacle when approached from the opposite direction. If the agent is on the one-way door square, then. with probability $p$, it moves through the door, no matter which action it executes. With probability $1-p$, however, the agent behaves as il it were on an empty square.

Consider the case where $p=0.75$. When following the expectation-optimal policy $\pi$ the agent has to go RIGHT in square $S$ and henee it moves to the one-way door square. 
where it has to select RIGHT again. The agent reaches its goal in the next step only with probability 0.25 because with probability 0.75 it falls through the one-way door. In the latter ease, it needs 18 additional steps to move around the vertical obstacle to the goal. Hence it needs $0.25 \cdot 2+0.75 \cdot 20=15.5$ steps on average to reach the goal from the start. The minimax-optimal policy suggests to go DOWN in square $S$ and move around the horizontal obstacle. This always takes 16 steps.

This example demonstrates that expectation-optimal policies do not necessarily have the lowest return in most cases. If, for example, the above experiment is repeated a large number of times then the expectation-optimal policy needs $25 \%$ more cost units in $75 \%$ of all cases when compared to the minimax-optimal policy. The latter policy minimizes the worst-case return and hence produces risk-avoiding behavior: the agent avoids the risk of falling through the onc-way door.

In the second example (Figure $1 \mathrm{~b}$ ), the vertical obstacle is eleven squares longer than in the previous example. Consider the case where $p-1 / 3$. Again, the expectation-optimal policy tells the agent to go across the one-way door because this requires on average $\frac{2 \cdot 2}{3}+\frac{42 \cdot 1}{3}-15 \frac{1}{3}<16$ steps. In this domain, the expectation-optimal policy has the lowest return (2 steps) in most cascs, but with probability $1 / 3$ the agent has to follow a very long path around the vertical obstacle. If the agent does not have enough cnergy for this long path (42 steps). it would be better to use the minimax optimal policy ( 16 steps).

This cxample demonstrates that the expected value criterion is not reliable, especially when solving tasks with resource constraints. Resource constraints cannot be addressed by giving the agent a big punishment (1.e., high immediate cost) when its accumulated cost has exceeded the given limit, because these reinforcement signals depend on the history of the agent's interaction with its environment and, consequently, do not satisfy the Markov property - unless the state representation is augmented with inlormation about the accumulated cost. This, however, would increase the size of the state space to an cxtent that makes efficient learning impossible. Fortunately, tasks for which the total cost is not allowed to exceed a given himit can be solved using the minimax criterion, because the minimax-optimal policy is guaranteed to obey this constraint if this is possible.

Recently, additional minimax-based reinforcement learning algorithms have been published. The model-based parti-game algorithm (Moore \& Atkeson, 1995) uses the minimax criterion for navigation tasks in conjunction with an adaptive state-action space represcntation. Using the minimax criterion has the advantage that it makes the planning atgorithm fast, allows it to use a simple world model, and automatically detects if a planning problem does not have a "safe" solution (in which case the partigame algorithm changes the state-action space). I ittman uses the minimax-criterion for Markov games (Littman, 1994) that have two kinds of uncertainties: the transition uncertainty (as commonly represented by MDPs) and, additionally, uncertainty introduced by an opponent who does not base his decisions on well defined stationary probabilities. To deal with the latter uncertainty, Littman uses the minimax criterion as is common in game theory.

Before we formalize and analyze the problem addressed in this article, we describe die deeny belind $Q$-luanting and Q-hat-leaning in Sections 3 and 4, respectively. First, 
however, we introduce our notation and give an overview of the commonly used RI. definitions and theorems.

\section{Basic Definitions and Theorems}

We define the set of admissible state-action pairs to be

$$
M:-\{(i, a) \in S \times A: a \in A(i)\}
$$

and call the elements of $\mathcal{R}^{M}$ state-action value functions or, synonymously, Q-functions. Assume that a function $Q \in \mathcal{R}^{M}$ and a state $i \in S^{Y}$ are given. Then, we call an action

$$
a \in A(i) \text { greedy for } i \text { and } Q \text { iff } a=\arg \min _{b \in A(i)} Q(i, b) \text {. }
$$

Furthermore, we call a stationary policy

$$
\pi \text { greedy for } Q \text { ifl } \forall i \in S: \pi(i)=\arg \min _{a \in \Lambda(i)} Q(i, a) \text {. }
$$

Finally, we call a tunction $Q \subset \mathcal{K}^{\text {ir }}$ greedy-equivalent to another function $Q^{\prime}=\mathcal{K}^{M}$ ift

$$
\{a \in A(i): a \text { is greedy for } i \text { and } Q\}=\left\{a \in A(i): a \text { is greedy for } i \text { and } Q^{\prime}\right\}
$$

for all $i \in S$. Let $c>0, i \in S, a \in A(i)$, and $Q \in \mathcal{R}^{M}$. We say that $a$ is 6 -greedy with respect to $i$ and $Q$ inf $Q(i, a)-\min _{b \in A(i)} Q(i, b) \mid<c$.

We define a partial order on $Q$-functions as follows: $Q \leq Q^{\prime}$ iff $Q(i, a) \leq Q^{\prime}(i, a)$ for all $i \in S$ and $a C A(i)$. For a given real number $r$ and a $Q$-function $Q$ we write $Q+r$ and $r \cdot Q$ to denote the $Q$-functions $Q^{\prime}$ and $Q^{\prime \prime}$ that satisfy $Q^{\prime}(i, a)-Q(i, a)+r$ and $Q^{\prime \prime}(i, a)-r \cdot Q(i, a)$, respectively, for all $i \in S$ and $a<A(i)$. We write $Q \leq r$ iff $Q(i, a)<r$ for all $i \in S$ and $a \in A(i)$. We use the maximum norm $\| \cdot i \mid$ for $Q$-functions, i.e.,

$$
|Q|=\max _{i c: s ; a \in A(i)}|Q(i, a)| .
$$

An element of $R^{\prime}$ is called a (state) value function. For a given real number $r$ and a value function $V$ we write $V-r$ and $r \cdot V$ to denote the value function $V^{\prime}$ and $V^{\prime \prime}$ that satisfy $V^{\prime}(i)-V^{\prime}(i)+r$ and $V^{\prime \prime}(i)=r \cdot V(i)$, respectively, for all $i c S$. We writc $V<r$ iff $V(i)<r$ for all $i \in S$. We use the maximum norm $\mid \cdot \|$ for value functions, i.e.,

$$
|V|:=\max _{i \in S}|V(i)|
$$

For a given Q-function $Q$ and stationary policy $r$ we define the value functions $V_{Q, \pi}$ and $V_{Q}$ lo bet

$$
\forall \in \in S: V_{Q, \pi}(i)-Q(i, \pi(i)) \text { and } V_{Q}(i)-\operatorname{minin}_{n(i)} Q(i, a)
$$

respectively.

In the following, we state six theorems that are used to prove the results of Section 5 The first three theorems follow immediately from the definitions of this section. 
THEOREm 1 Let $Q$ be a $Q$-function, $\pi$ a stationary policy, and $r \in \mathcal{R}$. Then, $V_{Q+r}-$ $V_{Q}+r$ and $V_{Q+r, \pi}=V_{Q, n}+r$

Thrmorem 2 Let $Q$ and $Q^{\prime}$ be $Q$-functions with $Q \leq Q^{\prime}$ and let $\pi$ be any stationary policy. Then, $V_{Q} \leq V_{Q^{\prime}}$ and $V_{Q, \pi} \leq V_{Q^{\prime}, \pi}$.

THEORFM 3 Let $\pi$ be a stationary policy and let $Q$ be a $Q$-function. Then $\pi$ is greedy for $Q$ iff $V_{Q, \pi}=V_{Q}$.

lhe following theorem is proven in (Willams \& Bard, 1993):

Trrmorrm 4 Let $g_{1}$ and $g_{2}$ be real-valued functions on a compact domain $U$. Then

$$
\left|\max _{u \in U} g_{1}(u)-\max _{u \in U} g_{2}(u)\right| \leq \max _{u \in U}\left|g_{1}(u)-g_{2}(u)\right| .
$$

THFORFM 5 Let $g_{1}$ and $g_{2}$ be real-valued functions on a compact domain $U$. Then

$$
\left|\min _{u \in U} g_{1}(u)-\min _{u \in U} g_{2}(u)\right| \leq \max _{u \in U}\left|g_{1}(u)-g_{2}(u)\right| .
$$

Proof: Let $u_{1}-\arg \min _{u \in U} g_{1}(u)$ and $u_{2}=\arg \min _{u \in U} g_{2}(u)$. Consider first the case where $g_{1}\left(u_{1}\right) \geq g_{2}\left(u_{2}\right)$. Then

$$
\begin{aligned}
\left|\min _{u \in \ell} g_{1}(u)-\min _{u \in U} g_{2}(u)\right| & -g_{1}\left(u_{1}\right)-g_{2}\left(u_{2}\right) \\
& \leq g_{1}\left(u_{2}\right)-g_{2}\left(u_{2}\right) \\
& \leq \max _{u \in V^{\prime}}\left|g_{1}(u)-g_{2}(u)\right| .
\end{aligned}
$$

A symmetrical argument establishes the same result for $q_{2}\left(u_{2}\right) \geq q_{1}\left(u_{1}\right)$.

ThHFORFM 6 for any stationary policy $\pi$ and arbitrary $Q$-functions $Q$ and $Q^{\prime}$ it holds that ||$V_{Q, \pi}-V_{Q^{\prime}, \pi} \mid<\left\|Q-Q^{\prime}\right\|$ and $\left\|V_{Q}-V_{Q^{\prime}}\left|\leq \| Q-Q^{\prime}\right|\right.$.

Proof: For any state $i$,

$$
: V_{C, \pi}(i) \quad V_{(2 ; \pi}(i)|=| Q(i, \pi(i)) \quad Q^{\prime}(i, \pi(i))|<| Q-Q^{\prime} \mid
$$

Since this holds for all states, the first inequality follows. For the second inequality, consider an arbitrary state: i Applying Thenrem 5 yields

$$
\begin{aligned}
& V_{Q}(i) \quad V_{Q^{\prime}}(i) \mid=\min _{\alpha \in A(i)} Q(i, a) \min _{a \in A(i)} Q^{\prime}(i, a) \\
& <\max _{a \in A(i)}\left|Q(i, a) \cdot Q^{\prime}(i, a)\right|<\| Q-Q^{\prime} \mid
\end{aligned}
$$

and the desired result follows. 


\section{Expectation-Based Markov Decision Tasks}

For expectation-based Markov decision tasks, one uses the value function $V_{\gamma}^{\pi} \in R^{S}$ to measure the performance of a given policy $\pi$ for a given MDP. The value function is defined as follows:

$$
\forall i \in S: \quad V_{\gamma}^{\pi}(i)=E\left(R_{\gamma}^{\pi} I_{0}^{\pi}=i\right)
$$

i.e., $V_{\gamma}^{\pi}(i)$ is the return one expects if the agent starts in state $i$ and uses policy $\pi$. A policy is called optimal if its value function is equal to the optimal value function $V_{\gamma}^{*} \in \mathcal{R}^{S}$ that is defined by

$$
V i \in S: V_{\gamma}^{*}(i)=\operatorname{ind}_{\pi} V_{\gamma}^{\pi}(i)
$$

\subsection{Dynamic Programming Operators}

A fundamental result from the theory of dynamic programming states that there always exists an optimal policy that is stationary. In dynamic programming, one approximates value functions of policies and optimal value functions by applying dynamic programming operators repeatedly. The dynamic programming operator $T_{\gamma}: \mathcal{R}^{S} \rightarrow \mathcal{R}^{S}$ is a mapping from value functions to value functions, defined by

$$
\forall V \in \mathcal{R}^{S} \forall i \in S: T_{\gamma} V(i)=\min _{a \in A(i)}\left[R(i, a)+\gamma \cdot \sum_{j \in S} P_{S}(i, a, j) V(j)\right]
$$

where $R(i, a)$ denotes the expected immediate cost for a given starting state $i$ and $\mathrm{ex}$ ecuted action $a^{1}$ For convenicnce we write $T_{\gamma} V(i)$ instead of $\left[T_{\gamma}(V)\right](i)$ and procecd similarly for other dynamic programming operators defined below. For a given stationary policy $\pi$, the dynamic programming operator $T_{\gamma}^{\pi}: \mathcal{R}^{S} \rightarrow \mathcal{R}^{S}$ is a mapping from value functions to value functions, defined by

$$
\forall V \subset \mathcal{R}^{S} \forall i \in S: T_{\gamma}^{\pi} V(i)=R(i, \pi(i))+\gamma \cdot \sum_{j \subset S} P_{S}(i, \pi(i), j) V(j)
$$

A policy is called greedy for a value function $V$ if

$$
\operatorname{Vi} \in S: \pi(i)=\arg \min _{a(i)}\left[R(i, a)+\gamma \cdot \sum_{j \in S} P_{S}(i, a, j) \cdot V(j)\right] \text {. }
$$

The following three theorems are known from the theory of dynamic programming (see, c.g., Bertsekas (1987)):

THEOREM 7 Let $V_{0} \in \mathcal{R}^{S}$ and $V_{k+1}=T_{\gamma} V_{k}$ for all $i \in S$ and $k \in \mathrm{N}$. Then $\lim _{k: \rightarrow \infty}\left|V_{k} \cdots V_{\gamma}^{*}\right|-0$. 
THEOREM 8 Let $\pi \in A^{S}$ be a stationary policy, $V_{0} \in \mathcal{R}^{S}$, and $V_{k+1}=T_{\gamma}^{\pi} V_{k}$ for all $i \in S$ and $k: \in \mathrm{N}$. Then $\lim _{k \rightarrow \infty}\left\|V_{k}-V_{\gamma}^{\pi}\right\|=0$.

THEOREM 9 A stationary policy $\pi$ is optimal iff it is greedy for $V_{\gamma}^{*}$.

Theorem 7 yields an algorithm for computing the optimal value function, and The orem 8 yields a similar algorithm for computing the value function of a given policy. Theorem 9 shows that optimal policies can be determined casily from a given optimal state value function. which is the reason why learning algorithms often approximate the optimal value function. To be able to compute a greedy policy for a given value function, one needs to know a world model, in this case the probabilities for the state transitions and the associated expected immediate costs

We conclude this section by defining the dynamic programming operators $B_{\gamma}$ and $B_{\gamma}^{\pi}$ for stationary policies $\pi$. These operators are associated with Q-functions and the expected-value criterion, and will be used in Section 5. $\mathcal{B}_{y}: \mathcal{R}^{M} \rightarrow \mathcal{R}^{M}$ satisfies

$$
B_{\gamma} Q(i, a)=R(i, a)+\gamma \cdot \sum_{j \in, S} P_{S}(i, a, j) V_{Q}(j)
$$

for any $Q$-function $Q$, state $i$, and action $a \in A(i) . B_{\gamma}^{\pi}: \mathcal{R}^{M} \ldots \mathcal{R}^{M}$ is defined by

$$
B_{\%}^{\pi} Q(i, a)-R(i, a)+\gamma \cdot \sum_{j \in S} P_{S}(i, a, j) V_{Q, \pi}(j)
$$

for any $Q$ function $Q$, state $i$, and action $a \in A(i)$.

In the next section. we introduce a reinforcement learning algorithm that learns a $Q$ function with the property that every policy that is greedy for this Q-function is optimal according to the expected value criterion. Greedy policies can be obtained from Qfunctions even if there is no world model available.

\subsection{Q-Learning}

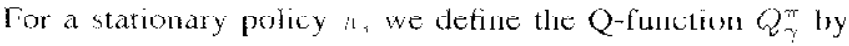

$$
Q(i, a)-R(i, a)+\gamma \cdot \sum_{j \in S} P_{S}(i, a, j) \cdot V_{\gamma}^{\pi}(j)
$$

for all is $S$ and $a \in A(i)$. The Q-learning algorithm (Watkins, 1989) approximates the optimal $Q$-function $Q *$ that is defined by

$$
Q_{(i, a)}-R(i, a)-\gamma \cdot \sum_{j \in S} P_{S}(i, a, j) \cdot V_{\%}^{*}(j)
$$

From Theorem 9, we conclude that a stationary policy is optimal iff it is greedy for $Q^{*}$. The $Q$-learning algorithm begins with an initial estimate $Q_{0}$ of $Q_{*}^{*}$ and improves this estinate as the Iesules of individual actigns become apparent. After each episode 
$t$, the Q-value associated with the starting state $i$ and executed action $a$ is updated as follows:

$$
Q_{t+1}(i, a)-\alpha_{t}(i, a)\left(r+\gamma V_{Q}(j)\right)+\left(1-\alpha_{l}(i, a)\right) Q_{t}(i, a)
$$

where $j$ is the successor state reached and $r$ is the immediate cost of the episode. The learning rates $\alpha_{t}(i, a)$ have to satisfy

$$
\sum_{i=0}^{\infty} \alpha_{l}(i, a)-\infty ; \quad \sum_{t=0}^{\infty} \alpha_{l}(i, a)^{2}<\infty .
$$

In (Watkins \& Dayan, 1992) and recently in (Tsitsiklis, 1994) it is proven that the Q-learning algorithm converges to $Q_{\gamma}^{*}$ if every action that is admissible in a state is executed infinitely often in that state.

\section{Minimax-Based Markov Decision Tasks}

The theory behind minimax-based Markov decision tasks is very similar to the one behind expectation-based tasks, as will become apparent in the following. We therefore use the same notation where appropriate, which also simplifies our presentation of a unified andalysis of both kinds of Markov decision tasks in a later section. From the context, it is always obvious which kind of task we are referring to.

The definitions in this section do not depend on the earlier definitions for expeclation-based Markov decision lasks, which avoids cyclic definitions. For mimimax-based Markov decision tasks, we use the value function $V_{\gamma}^{\pi} \in \mathcal{R}^{S}$ to measure the performance of a given policy $\pi$ for a given MDP. The value function is defined as follows:

$$
\forall i \in S: V_{\gamma}^{\pi}(i)=\sup \left\{r \in \mathcal{R}: P^{\prime}\left(R_{\gamma}^{\pi}>r \mid I_{0}^{\pi}=i\right)>0\right\}
$$

i.e., $V_{\gamma}^{\pi}(i)$ is the worst-case return that can possibly occur if the agent starts in state $i$ and uses policy $\pi$. A policy is called optimal if its value function is equal to the optimal value function $V_{y}^{*}<\mathcal{R}^{S}$ that is defined by

$$
\forall i \in S: \quad V_{\gamma}^{*}(l)-\inf _{\pi} V_{\gamma}^{\prime \prime}(i)
$$

\subsection{Dynamic Programming Uperators}

From (Heger, 1994b) we obtain the result that there always exists an optimal policy that is stationary. Let

$$
c(i, a, j)-\operatorname{sip}\left\{r \in C: P_{C}(i, a, j, r)>0\right\}
$$

denote the worstease immediate cost that can be obtaincd for the state transition from $i$ to $j$ under action $a$, and let

$$
N(i, a)-\left\{j<s^{y}: P_{S}(i, a, j)>0\right\}
$$


be the set of possible successor states when executing action $a$ in state $i$. The dynamic programming operator $T_{\gamma}: \mathcal{R}^{S} \rightarrow \mathcal{R}^{S}$ is a mapping from value functions to value functions, defined by

$$
\forall V \subset \mathcal{R}^{S} \forall i \in S: T_{\gamma} V(i)=\min _{a \in A(i)} \max _{j \in N(i, a)}[c(i, a, j)+\gamma \cdot V(j)] .
$$

For a given stationary policy $\pi$, the dynamic programming operator $T_{\gamma}^{\pi}: \mathcal{R}^{S} \rightarrow \mathcal{R}^{S}$ is a mapping from value functions to value functions, defined by

$$
\forall V \subset \mathcal{R}^{S} \forall i \in S: T_{\gamma}^{\pi} V(i)=\max _{j \in N(i, \pi(i))}[c(i, \pi(i), j)+\gamma \cdot V(j)] .
$$

A policy is called greedy for a value function $V$ if

$$
\forall i \in S^{\prime}: \pi(i)=\arg \min _{a \in A(i)}\left[\max _{j \in N(i, a)}(c(i, a, j)+\gamma \cdot V(j))\right] .
$$

The following thrce theorems are proven in (Heger, 1994b):

THEOREM 10 Let $\pi \in A^{S}$ be a stationary policy, $V_{0} \in \mathcal{R}^{S}$, and $V_{k+1}-T_{\gamma} V_{k}$ for all $i \in S$ and $k \in \mathrm{N}$. Then $\lim _{k+\infty}|| V_{k}-V_{\gamma}^{*} \mid i=0$.

THForfm 11 Let $\pi \subset \Lambda^{5}$ be a stationary policy, $V_{0} \in \mathcal{R}^{S}$, and $V_{k+1}=T_{\gamma}^{\pi} V_{k}$ for all $i \in S$ and $k \in N$. Then $\lim _{k \rightarrow \infty}\left\|V_{k} \cdots V_{\gamma}^{\pi}\right\|=0$.

THEOREM 12 A stationary policy $\pi$ is optimal iff it is greedy for $V_{\gamma}^{*-1}$.

The first two theorems yield algorithms for computing the optimal value function and the value function of a given policy. respectively. Theorem 12 shows that optimal policies can be determined easily from a given optimal value function. To be able to compute a greedy policy for a given value function, one needs to know a world model, in this case the sets of possible successor states for all state-action pairs and the worst immediate costs which the action executions can result in. Note that this world model is, in gencral, much simpler than the corresponding world model needed for expectation-based Markov decision tasks.

We conclude this section by defining the dynamic programming operators $B_{\gamma}$ and $B_{\gamma}^{\pi}$ for stationary policies $\pi$. These operators are associated with $\mathrm{Q}$-functions and the minimax criterion, and will he used in Sertion $5 R_{-y} \cdot \mathcal{R}^{M} \ldots \mathcal{R}^{M}$ sarisfics

$$
B_{\gamma} Q(i, a)-\max _{j \in N(i, a)}\left|c(i, a, j)+\gamma \cdot V_{Q}(j)\right|
$$

for any $Q$-function $Q$, state $i$, and action $a \in A(i) . B_{\gamma}^{\pi}: \mathcal{R}^{M} \rightarrow \mathcal{R}^{M}$ is defined by

$$
\left.B_{\gamma}^{\pi} Q(i, a)-\max _{j \in N(i, a)} c(i, a, j)+\gamma \cdot V_{Q ; \pi}(j)\right]
$$

for any $Q$-function $Q$, state $i$, and action $a \in A(i)$.

In the next section, we introduce a reinforcement learning algorithm that learns a $Q$ function with the property that every policy that is greedy for this Q function is optimal according to the minimax criterion. Its advantage is, as in the case of expectation-based Markov decision tasks, that one does not need to know a world model to compute a policy that is groedy for a given Q-function. 


\subsection{Q-Hat-Learning}

For a stationary policy $\pi$, we define the Q-function $Q_{\gamma}^{\pi}$ by

$$
Q_{\gamma}^{\pi}(i, a)=\max _{j \in N(i, a)}\left[c(i, a, j)+\gamma \cdot V_{\gamma}^{\pi}(j) !\right.
$$

for all $i \in S$ and $a \in A(i)$. The Q-hat-learning algorithm (Heger, 1994a) approximates the optimal $\mathrm{Q}$-function $Q_{\gamma}^{*}$ that is defined by

$$
Q_{\gamma}^{*}(i, a)=\max _{j \in N(i, a)}\left[c(i, a, j)+\gamma \cdot V_{\gamma}^{*}(j)\right]
$$

From Theorem 12. we conclude that a stationary policy is optimal iff it is greedy for $Q_{\gamma}^{*}$. The Q-hat-lcarning algorithm begins with an initial estimate $Q_{0} \leq Q_{\gamma}^{*}$ and improves this estimate as the results of individual actions become apparent. After each episode $t$, the $\mathrm{O}$-value associated with the starting state $i$ and executed action $a$ is updated as follows:

$$
Q_{t+1}(i, a)=\max \left\{Q_{t}(i, a) ; r+\gamma V_{Q_{t}}(j)\right\}
$$

where $j$ is the successor state reached and $r$ is the immediate cost of the episode. Note that the Q-hat-learning algorithm has no need for a learning rate, and that the Q-values are monotonely increasing in time. In (Heger, 1095) it is proven that the Q-hat learning algorithm converges to $Q_{\gamma}^{*}$ if every action that is admissible in a statc is executed infinitely often in that state.

An advantage of the Q-hat-learning algorithm is that the exploration-exploitation problem (c.g., Thrun, 1992) for Q-hat-learning is not as severe as the one for Q-lcarning: Let $\epsilon>0$ be a constant and assume that every state becomes infinitely often a starting state. Assume further that the agent always selects an action from the set of actions that are $\epsilon$-greedy with respect to its current $Q$-function and starting state, and that it selects an action from this set with uniform probability. Then, the Q-hat-learning algorithm converges, with probability one, to a $Q$-function $\hat{Q}$ that is greedy-equivalent to $Q_{\gamma}^{*}$, and $\hat{Q}(i, a)=Q_{\gamma}^{*}(i, a)$ with probability one for every state $i \in S$ and action $a \in A(i)$ that is greedy for $i$ and $Q_{\gamma}^{*}$.

\section{The Loss from Imperfect Value Functions}

In the previous two sections, we have scen that expectation-based and minimax-based tasks have in common that optimal policies can be determined by finding policies that are greedy for the optimal value function This is the reason why many dynamic programming approaches, such as the famous Q-learning algorithm and its counterpart for the minimax criterion, the Q-hat-learning algorithm, are only concerned with finding an optimal value function

The question remains to which extent the performance of the agent differs from its optimal performance if it uses a policy that is greedy for an approximation of the optimal value function instead of a policy that is greedy for the optimal value function itself; 
see for example (Singh \& Yee, 1994) and (Williams \& Baird, 1993). Knowing the magnitude of this loss in performance is important for at least two reasons: First dynamic programming and RL, algorithms itcratively improve an approximation of the optimal value function. The value function obtained after a finite number of itcrations is, in general, only an approximation of the optimal value function. Second, one cannot use lookup tables to represent value functions in complex domains, because there are too many states. This makes it necessary to use parametric function approximators, such as neural networks, that have the ability to generalize. Since function approximators cannot guarantec that they gencralize correctly, they can only be expected to approximate the optimal value function.

In practice, one can therefore only expect to obtain approximations of optimal value functions. If small deviations of these approximations from the optimal value functions resulted in an arbitrarily bad performance of the agent, this would raise significant concerns about the use of function approximators in dynamic programming-based learning.

\subsection{Results for Value Functions}

The maximum norm distance was used in (Singh \& Yec, 1994) to measure the approximation quality of a value function; in (Williams \& Baird, 1993), the so-called Bellman error magnitude was used for the same purpose. In both cases. the loss in performance turned out to be at most proportional to the approximation error for expectation-based Markov decision tasks.

We generalize this work by giving an abstract analysis that holds for both expectationbased tasks and minimax-based tasks. We only use properties that dynamic programming operators of both tasks have in common. In the following, we present these common fea tures as "assumptions" and defer their proof to Appendix A. Based on these assumptions, we present in this and the following section our results about the loss from imperfect value functions and Q-functions, respectively. The significance of these results will be discussed in the conclusion of this article, and the corresponding proofs can be found in Appendix B.

Assume $V_{\gamma}^{*}$ and $V_{\gamma}^{*}$ (for some stationary policy $\pi$ ) are value functions, and $T_{\gamma}$ and $T^{\prime r} \cdot \mathcal{R}^{S} \rightarrow \mathcal{R}^{S}$ are operatore These value functions and operators have to satisfy the following eight assumptions.

The first assumption guarantecs that the valuc function $V_{\gamma}^{\pi}\left(V_{\gamma}^{*}\right)$ is the only fixed point of the operator $\gamma_{\gamma}^{n}\left(T_{\gamma}\right)$

Assumption 1 Let $\pi$ be a stationary policy and $V$ be a value function. Then $T_{\gamma} V-V$ iff $V=V_{\gamma}^{\pi}$, and $T_{\gamma} V-V$ iff $V=V_{\gamma}^{*}$

Assume that we apply operator $T_{\gamma}$ or $T_{\gamma}^{\gamma}$ to a value function that is the sum of a real value $r$ and a value function $V$. The next assumption states that we obtain $\gamma \cdot r$ plus the value function that is obtained by applying the same operator to $V$.

Assumption 2 Let $\pi$ be a stationary policy, $V$ be a value function, and $r \in \mathcal{R}$. Then

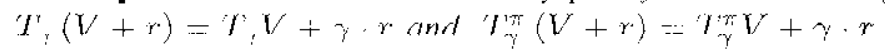


Third, we assume that both operators maintain the partial relation $\leq$ that was defined earlier for value functions.

Assumption 3 Let $V$ and $V$ be value functions with $U \leq V$ and $\pi$ be a stationary policy. Then $T_{\gamma} U<T_{\gamma} V$ and $T_{\gamma}^{\pi} U \leq T_{\gamma}^{\pi} V$.

Furthermore, we assume that the maximum norm distance between the images of any two value functions $U$ and $V$ is at most $\gamma$ times the maximum norm distance between $U$ and $V$, no matter which of the two operators is used.

Assumption 4 Let $\pi$ be a stationary policy and $U$ and $V$ be value functions. Then $\left\|T_{\gamma} U-T_{\gamma} V\right\| \leq \gamma \cdot\|U \quad V\|$ and $\left\|T_{\gamma}^{\pi} U-T_{\gamma}^{\pi} V\right\| \leq \gamma \cdot\|U-V\|$.

The next assumption defines greedy policies in an abstract way that does not depend on details of either expectation-based or minimax-based decision tasks. It also shows the fundamental relationship between the operators $T_{\gamma}^{\pi}$ and $T_{\gamma}$

Assumption 5 Let $\pi$ be any stationary policy and $V$ be any value function. Then $\pi$ is greedy for $V$ iff $T_{\gamma}^{\pi} V-T_{\gamma} V$.

The following assumption shows the fundamental relationship between the value functions $V_{\gamma}^{*}$ and $V_{\gamma}^{* r}$.

Assumption 6 Let i be any state and $\Pi_{S}$ be the set of stationary policies. Then $V_{\gamma}^{*}(i)=$ $\min _{\pi \in I_{S}} V_{\gamma}^{\pi}(i)$.

For deterministic MDPs, the starting state $i$ and the executed action a determine uniquely the successor state $j_{i}^{a}$ reached and the immediate cost $c_{i}^{a}$ obtained. We as. sume that for deterministic MDPs, the operators $T_{\gamma}$ and $T_{\gamma}^{\prime r}$ are defined in the following way.

Assumption 7 l.et $\pi$ be a stationary policy, $i$ be a state, and $V$ be a value function. Then $T_{\gamma} V(i) \cdot \min _{a \in A(i)}\left[c_{i}^{(n}+\gamma \cdot V\left(j_{i}^{n}\right)\right]$ and $T_{\gamma} V(i)-c_{i}^{\pi(i)}+\gamma \cdot V\left(j_{i}^{\pi(i)}\right)$ if the MDP is deterministic.

Consider a deterministic MDP, any stationary policy $\pi$, and a given starting state $i$ at time $t=0$. In this case the return, call it $r_{\gamma}^{\pi}(i)$, is uniquely determined. The eighth and final assumption states that $V_{\gamma}^{r}$ is equal to this value. ${ }^{2}$

Assumption $8 V_{7}^{\pi}(i) \cdots r_{\gamma}^{\pi}($ i) for ary deterministic Markov decision process, stationary policy $\pi$, and state $i$.

Before we can analyze the loss in performance due to imperfect value functions we have to define what we precisely mean by "imperfect." We measure the distance between a value function $V$ and the optimal value function $V_{*}^{*}$ in two different ways:

$$
\begin{aligned}
& d_{M}(V):-|| V \quad V_{\gamma}^{*}\left|: \cdots \max _{i \in S}\right| V(i) \cdots V_{\gamma}^{*}(i) \mid: \\
& d_{B}(V):-\left|T_{\gamma} V-V\right|:-\max _{\zeta} T_{\gamma} V(i)-V(a) \mid
\end{aligned}
$$


We call $d_{M}(V)$ the maximum norm distance between $V$ and $V_{\gamma}^{*}$ and, following Williams and Baird, $d_{B}(V)$ the Bellman error magnitude. From its definition it is not obvious that the Bellman error magnitude is a reasonable measure for the distanec betwecn $V$ and $V_{\gamma}^{*}$. The next two theorems show that this is indeed the case.

THAORR A.M 13 f et $V$ he any value function and $\pi$ be any stationary policy. Then

$$
\begin{aligned}
& (1-\gamma) \cdot\left\|V-V_{\gamma}^{*}\right\|<\left\|V-T_{\gamma} V\right\| \leq(1+\gamma) \cdot\left\|V \cdot V_{\gamma}^{*}\right\| \\
& (1-\gamma) \cdot\left\|V-V_{\gamma}^{\pi}\right\| \leq\left\|\left.V \cdots T_{\gamma}^{7} V\right|_{1} ^{\vdots} \leq(1+\gamma) \cdot\right\| V-V_{\gamma}^{\pi} \|_{1} .
\end{aligned}
$$

I'HEOREM 14 Let $V$ be a value function that satisfies $V \leq V_{\gamma}^{*}$ and $\pi$ be any stationary policy. Then

$$
\begin{aligned}
& (1-\gamma) \cdot\left\|V-V_{\gamma}^{*}\right\| \leq\left\|V-T_{\gamma} V\right\| \leq\left\|V-V_{\gamma}^{*}\right\| ; \\
& (1-\gamma) \cdot\left\|V-V_{\gamma}^{*}\right\|<\left\|V-T_{\gamma}^{* \pi} V\right\| \leq\left\|V-V_{\gamma}^{\pi \pi}\right\| .
\end{aligned}
$$

Theorem 13 ensures that $(1-\gamma) \cdot d_{M}(V)<d_{B}(V) \leq(1+\gamma) \cdot d_{M}(V)$. This tight relationship between the Bellman error magnitude and the maximum norm distance justifics the use of the Bellman error magnitude as a measure for the distance between $V$ and $V_{\gamma}^{*}$. Its advantage over the maximum norm distance is that it can be computed easily even if $V_{\gamma}^{*}$ is unknown.

We measure the loss in performance due to a policy $\pi$ by $\left\|V_{\gamma}^{\pi}-V_{\gamma}^{*}\right\|$. The following two theorems guarantee that the loss in performance is small if dynamic programming based learning approaches are used that satisfy the assumptions above, good approximations of the optimal value functions are achieved, and then a greedy policy is foltowed - provided that the discount factor $\gamma$ is not 100 close to 1.0 .

THEOREM 15 Let $V$ be a value function and $\pi$ be a stationary policy that is greedy for $V$ Then $\left|V_{\gamma}^{\pi}-V_{\gamma}^{*}\right| \leq \frac{2 \gamma}{1-\cdots} \cdot d_{t s}(V)$. Furthermore, this bound is tight, i.e, there exists

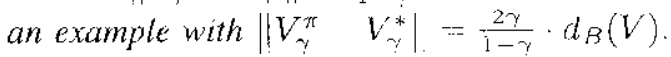

TIIEOREM 16 Let $V$ be a value function and $\pi$ be a stationary policy that is greedy for $V$. Then ||$V_{\gamma}^{r}-V_{\gamma} \mid<\frac{i_{\gamma}}{k} \cdot a_{M}(V)$. Furthermore, this bound is tight.

The next theorem and corollary guarantee that the bound for the loss in performance can he reduced by a factor of 2 if the approximation $V$ underestimates $V_{\gamma}^{*}$.

'THEOREM 17 Let $V$ be a value function with $V \leq V_{\gamma}^{*}$ and $\pi$ be a stationary policy that is greedy for $V$. Then ||$V_{\gamma}^{*} \quad V_{\gamma}^{*} \mid \leq \frac{\gamma}{1-\gamma} \cdot d_{\ell}(V)$. Furthermore, this bound is tight.

COROLLARY 1 Let $V$ be a value function with $V \leq V_{\gamma}^{*}$ and $\pi$ be a stationary policy that is greedy for $V$. Then ||$V_{*}^{*}-V_{3}^{*} \mid<\frac{\gamma}{1-\gamma} \cdot d_{M}(V)$. Furthermore, this bound is tight.

It might not always be easy to verify that $V<V_{*}^{*}$ if $V_{\gamma}^{*}$ is unknown, but the following two corollaries provide a sufficient condition for $V \leqslant V_{\gamma}^{*}$ that depends only on the dynamic programming operator $Y_{Y}$ and is easy to verify. 
COROLLARY 2 Let $V$ be a value function and $\pi$ be a greedy policy for $V$. If $V \leq T_{\gamma} V$, then $\left\|V_{\gamma}^{\pi}-V_{\gamma}^{*}\right\| \leq \frac{\gamma}{1-\gamma} \cdot d_{B}(V)$. Furthermore, this bound is tight.

COROLLARY 3 Let $V$ be a value function and $\pi$ be a greedy policy for $V$. If $V \leq T_{\gamma} V$, then $\left\|V_{\gamma}^{\pi} \cdot V_{\gamma}^{*}\right\| \leq \frac{\gamma}{1-\gamma} \cdot d_{M}(V)$. Furthermore, this bound is tight.

\subsection{Results for Q-Functions}

This section proceeds similarly to the last section, except that our results now apply to Qfunctions instead of value functions: We first present features of dynamic programming operators that hold for both expectation-based and minimax-based Markov decision tasks and then derive theorems based on these assumptions

Assume that $V_{\gamma}^{*}$ and $V_{\gamma}^{\pi}$ (for some stationary policy $\pi$ ) are value functions, $Q_{\gamma}^{*}$ and $Q_{\gamma}^{\pi}$ are Q-fiunctions, and $B_{\gamma} \cdot \mathrm{R}^{M} \rightarrow \mathcal{R}^{M}$ and $B_{\gamma}^{\pi}: \mathcal{R}^{M} ; \mathcal{R}^{M}$ are operators. These value functions, Q functions, and operators have to satisfy the following assumptions in addition to the eight assumptions stated in the previous section. We show in Appendix A that all of thece xceumptions are satisfied for both expectation-based and minimax-based Markov decision tasks.

Assumption 9 relates the value functions $V_{\gamma}^{*}$ and $V_{\gamma}^{\pi}$ to their corresponding Q-functions $Q^{*}$, and $Q_{\gamma}^{\pi}$, respectively

Assumption 9 Let $\pi$ be a stationary policy. Then $V_{Q ;}=V_{\gamma}^{*}$ and $V_{Q_{\gamma}^{\pi}, \pi}=V_{\gamma}^{\pi}$.

Assumption 10 guarantees that the Q-function $Q_{\gamma}^{\pi}\left(Q_{\gamma}^{*}\right)$ is the only fixed point of the operator $B_{\gamma}^{\pi}\left(B_{\gamma}\right)$.

Assumption 10 Let $\pi$ be a stationary policy and $Q$ be a $Q$-function. Then $B_{\gamma} Q=Q$ iff $Q=Q_{\gamma}^{*}$ and $B_{\gamma}^{\pi} Q=Q$ iff $Q-Q_{\gamma}^{\pi}$.

Assume that we apply operator $B_{\gamma}$ or $B_{\gamma}^{\pi}$ to a $\mathrm{Q}$-function that is the sum of a real value $r$ and a $Q$-function $Q$. Assumption 11 states that we obtain $\gamma \cdot r$ plus the $Q$ function that is obtained by applying the same operator to $Q$.

Assumption 11 Let $\pi$ be a stationary policy, $Q$ be a $Q$-function, and $r \mathcal{R}$. Then $B_{\gamma}(Q+r)=B_{\gamma} Q+\gamma \cdot r$ and $B_{\gamma}^{\pi}(Q+r)=B_{\gamma}^{\pi} Q+\gamma \cdot r$.

Assumption 12 states that both operators maintain the partial relation $<$ for $Q$-functions.

Assumption 12 Let $Q$ and $Q^{\prime}$ be $Q$ functions with $Q<Q^{\prime}$ and $\pi$ be a stationary policy. Then $B_{\gamma} Q \leq B_{\gamma} Q^{\prime}$ and $B_{\gamma}^{\prime \prime} Q<B_{\gamma}^{\pi} Q^{\prime}$.

We assume that the maximum norm distance between the images of any two Qfunctions $Q$ and $Q^{\prime}$ is at most $\gamma$ times the maximum norm distance between $Q$ and $Q^{\prime}$, no matter which of the two operators is used

Assumption 13 Let $\pi$ be a stationary policy and $Q$ and $Q^{\prime}$ be $Q$-functions. Then $\left\|B_{\gamma} Q \cdots B_{\gamma} Q^{\prime}\left|\leq \gamma \cdot \| Q \cdot Q^{\prime}\right|\right.$ and $\| B_{\gamma}^{\pi} Q-B_{\gamma}^{*} Q^{\prime}\left|<\gamma \cdot \| Q \cdots Q^{\prime}\right|$.

Assumption 14 shows the fundamental relationship belween the operators $F_{\gamma}^{* *}$ and $B_{\gamma}$. 
Assumption 14 Let $Q$ be a $Q$-function and $\pi$ be a stationary policy that is greedy for Q. Then $B_{\gamma} Q=B_{\gamma}^{\pi} Q$.

Assumption 15 shows the fundamental relationship between the $Q$-functions $Q_{\gamma}^{*}$ and $Q_{\gamma}^{3 T}$

Assumption 15 Let $i b c$ a state, a $<A(i)$, and $\mathrm{II}_{5}$ bo the set of stationary policies. Then $Q_{\gamma}^{*}(i, a)-\min _{\pi \in \Pi,} Q_{\gamma}^{\pi}(i, a)$.

The following assumption gives the definitions of the operators $B_{\gamma}$ and $B_{\gamma}^{\pi}$ for deterministic MDPs.

Assumption 16 Let $\pi$ be a stationary policy, $i$ be a state, $a \in A(i)$ be an action, and $Q$ be a $Q$-function. Then $D_{\gamma} Q(i, a)-c_{i}+\gamma \cdot V_{Q}\left(j_{i}^{a}\right)$ and similarly, $B_{\gamma}^{\pi} Q(i, a)-$ $a_{i}^{a}+\gamma \cdot V_{Q, \pi}\left(j_{i}^{a}\right)$ if the MDP is deterministic.

The final assumption relates the definition of an optimal policy to the $\mathrm{Q}$-function $Q_{\gamma}^{*}$.

Assumption 17 A stationary policy is optimal iff it is greedy for $Q_{\gamma}^{*}$

Belore we can analyze the loss in performance due to imperfect Q-functions we have to definc what we precisely mean by "imperfect." We measure the distance between a Q-function $Q$ and the optimal Q-function $Q_{\gamma}^{*}$ in two different ways:

$$
\begin{aligned}
& a_{M}(Q):-\left\|Q-Q_{\gamma}^{+}\right\|=\max _{i \in S, a \in A(i)} Q(i, a)-Q_{\gamma}^{+}(i, a) ; \\
& d_{B}(Q):=\left\|B_{\gamma} Q \quad Q\right\|=\max _{i \in S, a \in A(i)}\left|B_{\gamma} Q(i, a) \quad Q(i, a)\right| .
\end{aligned}
$$

We call $d_{M}(Q)$ the maximum norm distance between $Q$ and $Q_{-}^{*}$ and, following Williams and Baird (Williams \& Baird, 1993), $d_{B}(Q)$ the Bellman error magnitude.

THEOREM 18 Let $Q$ be a function and $\pi$ be a stationary policy. Then

$$
\begin{aligned}
& (1-\gamma) \cdot\left\|Q-Q_{\gamma}^{*} \mid \leq\right\| Q-B_{\gamma} Q\|\leq(1+\gamma) \cdot\| Q-Q_{\gamma}^{*} \| ; \\
& (1-\gamma) \cdot j\left|Q \cdot Q_{\gamma}^{\pi}\right||<| Q-I 3_{\gamma}^{\pi} Q|<(1+\gamma) \cdot| Q-Q_{-\gamma}^{\pi} \mid i .
\end{aligned}
$$

IHFOREM 19 Let $Q$ be a $O$-function with $Q<Q^{*}$ and $\pi$ be a stamionary policy. Then

$$
\begin{aligned}
& \left(\begin{array}{ll}
1 & \gamma
\end{array}\right) \cdot\left|Q-Q_{\gamma}^{* !}\right| \leq\left|Q-B_{\gamma} Q\right| \leq\left|Q-Q_{\gamma}^{*}\right| ; \\
& \left(\begin{array}{ll}
1 & y
\end{array}\right) \cdot\left|Q-Q_{r}^{\pi}\right|_{i}^{i}<|| Q-B_{\gamma}^{*} Q !<\left|Q-Q_{y}^{*}\right| !
\end{aligned}
$$

Theorom 18 ensures that $(1-\gamma) \cdot d_{w}(Q) \leq d_{B}(Q) \leq(1+\gamma) \cdot d_{M}(Q)$. This relationship justifies to use the Bellman error magnitude as a measure for the distance between $Q$ and $Q_{\sim}^{*}$. Its advantage over the maximum norm distance is that it can be computed easily even if $Q^{*}$ is unknown.

The following rwo theorems guarantee that the loss in performance is small if dynamic programming based learning approaches are used that satisfy the assumptions above. have achieved good approximations of the optimal Q-function, and then follow a greedy policy - provided that the discount bator $\gamma$ is not ion close in 10 
THForFM 20 Let $Q$ be a $Q$-function and $\pi$ be a stationary policy that is greedy for $Q$. Then $\left\|V_{\gamma}^{\pi}-V_{\gamma}^{*}\right\|<\frac{2}{1-\gamma} \cdot d_{B}(Q)$. Furthermore, this bound is tight.

THEOREM 21 Let $Q$ be a Q-function and $\pi$ be a stationary policy that is greedy for $Q$. Then ||$V_{\gamma}^{\pi}-V_{\gamma}^{*} \mid \leq \frac{2}{1-\gamma} \cdot d_{M}(Q)$. Furthermore, this bound is tight.

The next theorem and corollary guarantee that the bound for the loss in performance can be reduced by a factor of 2 if the approximation $Q$ underestimates $Q_{\gamma}^{*}$. These results apply especially to Q-hat-learning because this algorithm operates with underestimations of the minimax-optimal Q-function.

Trmor.am 22 Let $Q$ be a $Q$ function with $Q \leq Q_{\gamma}^{*}$ and $\pi$ be a stationary policy that is greedy for $Q$. Then $\left\|V_{\gamma}^{\pi}-V_{\gamma}^{*}\right\| \leq \frac{1}{1-\gamma} \cdot d_{B}(Q)$. Furthermore, this bound is tight.

COROLLARY 4 Let $Q$ be a $Q$-finction with $Q \leq Q_{\gamma}^{+}$and $\pi$ be a stationary polvcy that is greedy for $Q$. Then $\left\|V_{\gamma}^{\pi}-V_{\gamma}^{*}\right\| \leq \frac{1}{1-\gamma} \cdot d_{M}(Q)$. Furthermore, this bound is tight.

It might not always be easy to verify that $Q_{\mathcal{C}} \leq Q_{\gamma}^{*}$ if $Q_{\gamma}^{*}$ is unknown, but the following two corollaries provide a sufficient condition for $Q \leq Q_{\gamma}^{*}$ that depends only on the dynamic programming operator $B_{\gamma}$ and is casy to verify.

COROLlary 5 Let $Q$ be a $Q$-function with $Q \leq B_{\gamma} Q$ and $\pi$ be a stationary policy that is greedy for $Q$. Then $\left\|V_{\gamma}^{\pi}-V_{\gamma}^{*}\right\| \leq \frac{1}{1-\gamma} \cdot d_{B}(Q)$. Furthermore, this bound is tight.

COROLIARY 6 Let $Q$ be a $Q$-function with $Q<B_{\gamma} Q$ and $\pi$ be a stationary policy that is greedy for $Q$. Then $\left\|V_{\gamma}^{\pi}-V_{\gamma}^{*}\right\| \leq \frac{1}{1-\gamma} \cdot d_{M}(Q)$. Furthermore, this bound is tight.

The following theorem and corollary show that policies that are greedy for $\mathrm{Q}$-functions are optimal if the Q-functions approximate the optimal Q-function closely.

THEOREM 23 There exists an $t>0$ with the following property: Every stationary policy is optimal if it is greedy for a $Q$-function $Q$ with $d_{M}(Q)<c$.

COROLLARY 7 There exists an $6>0$ with the following property: Every stationary policy is optimal if it is greedy for a $Q$-function $Q$ with $d_{B}(Q)<t$.

However, Q-functions that do not approximate the optimal Q-function well do not necessarily prevent one from obtaining optimal policies, as the following two results show.

THEOREM 24 for every real number $r$ there exists a $Q$ function $Q$ with $d_{M}(Q)>r$ that has the following property: Every stationary policy that is greedy for $Q$ is optimal.

COROLLARY 8 for every real number $r$ there exists a $Q$-function $Q$ with $d_{B}(Q)>r$ that has the following property: Every stationary policy that is greedy for $Q$ is optimal. 


\section{Conclusions}

In this paper, we have studicd both minimax-based and expectation-based Markov decision tasks and quantified how much performance is lost if one uses value functions that approximate the optimal value function instead of the optimal value function itself. In particular, we described the properties that both Markov decision tasks have in common (namely Assumptions 1 to 17) and based our analysis solely on these properties. Using this framework, we were able to transfer the results of (Williams \& Baird, 1993) and (Singh \& Yee, 1994) to minimax-based Markov decision tasks. To the best of our knowledge, our analysis is the first one that studies approximate value functions for minimax-based Markov decision tasks and the resulting loss in performance.

Our main result is that this loss in performance is at most proportional to the approximation error (Theorems 15, 16, 20 and 21). The factor of proportionality, however, depends on the discount factor $\gamma$ and approaches infinity if $\gamma$ approaches one. Unfortunately, there are some Markov decision tasks with delayed reinforcement for which it is not reliable to choose $\gamma$ freely (Schwart7, 1993; see also McDonald \& Hingson, 1994).

We obtained previously unknown results that hold for both the expectation-based tasks and the minimax-based tasks. The right hand side inequalities of the Theorems 13, 14, 18 , and 19 we proved show that the Bellman error magnitude (used in (Williams \& Baird. 1993) in order to measure the quality of approximation) is more related to the maximum norm distance (used in (Singh \& Yee, 1994)) than was previously known. The results were useful to derive new bounds. Furthermore, on the one hand, we showed that the bounds given in (Singh \& Yce, 1994) are tight, i.e., there are examples where these bounds are attained (Theorem 16 and 21). On the other hand if one assumes $V \leq V_{\gamma}^{*}$ or $Q \leq Q_{\gamma}^{*}$ then the corresponding bounds can be reduced by a factor of two. These new bounds were also proven to be tight (Corollaries 1 and 4).

In the Q-hat-learning algorithm, the Q-function $Q$ always satisfies $Q \leq Q_{\gamma}^{*}$ with probability onc. This is generally not true in $Q$-learning. Hence the results of Theorem 22 and Corollary 4 are especially important in minimax-hased tasks

We showed that small non-zero approximation crrors in Q-functions are already sufficient to obtain optimal policies (Theorem 23 and Corollary 7). As a consequence, every algorithm that iteratively produces a sequence of Q-functions that converges in infinity to the optimal Q-function, is able to determine optimal policies after a finite time. On the other hand we also showed that small approximation errors are not necessary to obtain optimal policies (Theorem 24 and Corollary 8).

It is still an open problem why expectation-based and minimax-based Markov decision tasks share so many properties. Another related open problem is whether there are other types of Markov decision tasks that satisfy our assumptions. Answers to these questions would probably lead to a better understanding of the existing reinforcement learning algorithms and might lead to the development of algorithms that use decision criteria llat ane differcin fion the ones we use today.

We have seen that the loss in performance is small when acting greedily on imperfect value functions if the value functions approximate the optimal value function well. A related issuc is whether the reinforcement learning atgorithms that were designed to be 
used in conjunction with lookup tables work equally well with function approximators. Theoretical results can be found in (Thrun \& Schwartz, 1993), (Baird, 1995), and (Gordon, 1995). Practical results in using RL algorithms with function approximators are published in (Tesauro, 1992), (Lin, 1993), (Crites \& Barto, 1995), (Boyan \& Moore, 1995), (Zhang \& Dietterich, 1995), and (Sutton, 1995). RL algorithms that are specifically designed to be used with function approximators were recently published in (Baird, 1995) and (Boyan \& Moore 1995).

\section{Acknowledgments}

Many spocial thanks to Suen Knenig for his suhctantial holp with the final editing of this. article.

\section{Appendix A}

\section{Proofs of the Assumptions}

It is easy to see that the seventeen assumptions hold for expectation-based Markov decision tasks. They are either standard results from dynamic programming (see e.g., Bertsekas, 1987; Ross, 1970) or follow immediately from Section 3. Assumptions 9 and 10 were proven in (Williams \& Baird, 1993).

The seventeen assumptions also hold for minimax-based Markov decision tasks. Assumption 1 was proven in (Heger, 1994b). We prove $\Lambda$ ssumptions 4, 9, 10, and 13 in the following. The remaining eleven assumptions then follow immediately from the results of Section 4.

\section{Proof of Assumption 4 for Minimax-Based Markov Decision Tasks:}

Assume $T_{\gamma} V\left(i^{*}\right) \geq T_{\gamma} V\left(i^{*}\right)$ for $i^{*}=\arg \max _{i \in S} T_{\gamma} V(i) \quad T_{\gamma} V(i)$ : and let $a^{*}=$ $\left.\arg \min _{a \in \Lambda\left(i^{*}\right)} \max _{j \in N\left(i^{*}, a\right)}\left[c\left(i^{*}, a, j\right) \mid \gamma V(j)\right]\right]$. Then, according to Theorem 4 ,

$$
\begin{aligned}
& \left\|T_{\gamma} U \quad T_{\gamma} V\right\|-\left|T_{\gamma} V^{\gamma}\left(i^{*}\right)-T_{\gamma} V\left(i^{*}\right)\right| \\
& -T_{\gamma} U\left(i^{*}\right)-T_{\gamma} V\left(i^{*}\right) \\
& =\min _{a \in A(i, j), j \in N\left(i^{*}, a\right)}\left[c\left(i^{*}, a, j\right) \mid \gamma U(j)\right]
\end{aligned}
$$

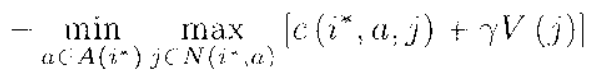

$$
\begin{aligned}
& <\max _{j\left(i^{*}, a^{*}\right)}\left[a\left(i^{*}, a^{*}, j\right)+\gamma^{\prime}(j) \vdots\right. \\
& -\max _{j \in N\left(i^{*} \cdot a^{*}\right)}\left[c\left(i^{*}: a^{*}: j\right)+\gamma V(j)\right] \\
& <\max _{j \in N\left(i^{*}, a^{*}\right)}\left|c\left(i^{*}, a^{*}, j\right)+\gamma U(j)-\left(c\left(i^{*}, a^{*}, j\right)+\gamma V(j)\right)\right| \\
& =\gamma \cdot \max _{\left.j \in \omega_{(i \cdots *}\right)}|U(j)-V(j)|<\gamma \cdot\|U-V\| .
\end{aligned}
$$


A symmetrical arguments holds if $T_{\gamma} V\left(i^{*}\right) \geq T_{\gamma}^{\prime} U\left(i^{*}\right)$. This proves the first inequality. To prove the second inequality, first consider the special case where $A(i)=\{\pi(i)\}$ for all $i \in S$. In this case, $T_{\gamma}^{\prime}-T_{\gamma}^{\pi r}$ (see (1) and (2) at page 206) and the sceond incquality follows from the first one. To show that the second inequality holds in general, notice that it does not depend on any assumptions about the sets $A(i)$ other than $\pi(i) \in A(i)$, which is trivially true.

Proof of Assumption 9 for Minimax-Based Markov Decision Tasks: According to Assumption 1, which was proven in (Heger 1994b), it holds for any state $i$ that

$$
\begin{aligned}
V_{Q_{\gamma}}(i) & =\min _{a \in A(i)} Q_{\gamma}^{*}(i, a)=\min _{a \in A(i)} \max _{j \in N(i, a)}\left[c(i, a, j)+\gamma \cdot V_{\gamma}^{*}(j)\right] \\
& =T_{\gamma} V_{\gamma}^{*}(i)=V_{\gamma}^{*}(i)
\end{aligned}
$$

which proves the first equation. Similarly, it follows from Assumption I that

$$
\begin{aligned}
V_{Q_{\gamma} ; \pi}(i)= & Q_{\gamma}^{\pi}(i, \pi(i))=\max _{j \in N(i, \pi(i))}\left[c(i, \pi(i), j)+\gamma \cdot V_{\gamma}^{\pi}(j)\right] \\
\cdots & T_{\gamma}^{\pi} V_{\gamma}^{\pi}(i)=V_{\gamma}^{\pi}(i)
\end{aligned}
$$

which proves the second equation.

Proof of Assumption 10 for Minimax-Based Markov Decision Tasks: According to Assurnption 9 , it holds for any state $i$ and action $a<A(i)$ that

$$
\begin{aligned}
B_{\gamma} Q_{\gamma}^{*}(i, a) & =\max _{j \in N(i, a)}\left[c(i, a, j)+\gamma \cdot V_{\left.Q_{\gamma}^{*}(j)\right]}\right. \\
& =\max _{j \in N(i, a)}\left[c(i, a, j)+\gamma \cdot V_{\gamma}^{*}(j)\right]-Q_{\gamma}^{*}(i, a)
\end{aligned}
$$

which proves the first equation. Similarly, if follows from Assumption 9 that

$$
\begin{aligned}
B_{\gamma}^{\pi} Q_{\gamma}^{\pi}(i, a) & =\max _{j \in N(i, a)}\left[c(i, a, j)+\gamma \cdot V_{Q *, \cdots}(j)\right] \\
& -\max _{j \in N(i, a)}\left[c(i, a, j)+\gamma \cdot V_{\gamma}^{\pi}(j)\right]-Q_{\gamma}^{\pi}(i, a)
\end{aligned}
$$

which proves the second equation.

Proof of Assumption 13 for Minimax-Based Markov Decision Tasks: Let $i \in S$ and $a \in A(i)$. From Theorems 4 and 6 we can conclude

$$
\begin{aligned}
& || B_{\gamma}^{\pi} Q-B_{\gamma}^{\pi} Q^{\prime}|| \\
\leq & \left|B_{\gamma}^{\pi} Q(i, a) \cdot B_{\gamma}^{\pi} Q^{\prime}(i, a)\right| \\
- & \left.\left|\max _{\mid \gamma \in \mathbb{N}(\gamma, a)}\right| c(i, a, j)+\gamma \cdot V_{Q, \pi}(j)\right]-\max _{j=N(i, a)}\left|c(i, a, j)+\gamma \cdot V_{Q^{\prime}, \pi}(j) !\right|
\end{aligned}
$$




$$
\begin{aligned}
& \leq \max _{j \in N(i, a)}\left|c(i, a, j)+\gamma \cdot V_{Q, \pi}(j)-\left(c(i, a, j)+\gamma \cdot V_{Q^{\prime}, \pi}(j)\right)\right| \\
& =\gamma \cdot \max _{j \in N(i, a)}\left|V_{Q, \pi}(j)-V_{Q^{\prime}, \pi}(j)\right| \\
& \leq \gamma \cdot\left\|V_{Q, \pi}-V_{Q^{\prime}, \pi}\right\| \\
& <\gamma \cdot\left\|Q \cdot Q^{\prime}\right\| .
\end{aligned}
$$

This proves the second inequality. The proof of the first inequality is obtained from this proof by substituting $B_{\gamma}, V_{Q}$, and $V_{Q^{\prime}}$ for $B_{\gamma^{\prime}}^{\pi}, V_{Q, \pi}$, and $V_{Q^{\prime}, \pi}$, respectively.

\section{Appendix B}

\section{Proofs of the Results from Section 5}

Proof of Theorem 13: The following four inequalities follow from Assumptions 1 and 4 in conjunction with the triangle inequality.

$$
\begin{aligned}
& \left|i V-V_{\gamma}^{*}\right| \vdots<\left\|V \quad T_{\gamma} V\right\|+\left\|T_{\gamma} V-V_{\gamma}^{*}\right\| \leq\left\|V \cdots T_{\gamma}^{\gamma} V\right\|+\gamma \cdot \| V-V_{\gamma}^{*} \mid ; \\
& \left\|V-T_{\gamma} V\left|i<\left\|V-V_{\gamma}^{*}\right\|+\left\|V_{\gamma}^{*}-T_{\gamma} V\right\|<\left\|V-V_{\gamma}^{*}\right\|+\gamma \cdot\right| V_{\gamma}^{*}-V\right\| ; \\
& \left\|V-V_{\gamma}^{\pi}\right\| \leq\left\|V \quad T_{\gamma}^{\pi} V\right\|+\left\|T_{\gamma}^{\pi} V-V_{\gamma}^{\pi}\right\| \leq\left\|V \quad T_{\gamma}^{\pi} V !+\gamma \cdot\right\| V-V_{\gamma}^{\pi} \mid \\
& \left\|V-T_{\gamma}^{\pi} V\right\|<\left\|V-V_{\gamma}^{\pi} \mid i+\right\| V_{\gamma}^{\pi}-T_{\gamma}^{\pi} V\|<\| V \quad V_{\gamma}^{\pi}\|+\gamma \cdot\| V_{\gamma}^{\pi}-V \| .
\end{aligned}
$$

Inequalities (B. 1), (B.2), (B.3), and (B.4) imply the first, second, third, and fourth inequality of this theorem, respectively.

Proof of Theorem 14: The first and third inequality have already been proven in the context of Theorem 13. From Assumptions 3, 1 , and 6 we obtain

$$
\begin{gathered}
T_{\gamma} V \quad\left\|V \quad \cdot V_{\gamma}^{*}\right\| \leq T_{\gamma}^{*} V_{\gamma}^{*}-\left\|V-V_{\gamma}^{*}\right\|_{i}^{i}=V_{\gamma}^{*} \cdots\left\|V-V_{\gamma}^{*}\right\| \leq V ; \\
T_{\gamma}^{\pi} V-\left\|V \cdot V_{\gamma}^{\pi} \mid \leq T_{\gamma}^{\pi} V_{\gamma}^{\pi}-\right\| V-V_{\gamma}^{\pi}\left\|-V_{\gamma}^{\pi} \quad\right\| V \quad V_{\gamma}^{\pi} \| \leq V .
\end{gathered}
$$

Assumptions 1 and 4 imply

$$
\begin{aligned}
& \left\|V_{\gamma}^{*} \quad T_{\gamma} V:\left|-\left\|T_{\gamma} V_{\gamma}^{*}-T_{\gamma} V\right\|<\gamma \cdot\left\|\left.V_{\gamma}^{*} \quad V\right|_{i} \leq\right\| V-V_{\gamma}^{*} \| ;\right.\right.
\end{aligned}
$$

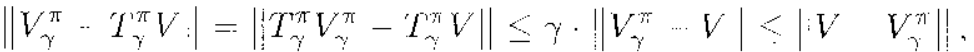

and therefore, in conjunction with Assumption 6,

$$
\begin{array}{ccc}
V \quad T_{\gamma} V \leq V_{\gamma}^{*}-T_{\gamma} V<\left\|V_{\gamma}^{*} \quad T_{\gamma} V\right\| \leq\left\|V \quad . V_{\gamma}^{*}\right\| ; \\
V \quad T_{\gamma}^{\pi} V \leq V_{\gamma}^{\pi}-T_{\gamma}^{\pi} V<\left\|V_{\gamma}^{\pi} \quad T_{\gamma}^{\pi} V\right\| \leq \| V-V_{\gamma}^{\pi} \mid
\end{array}
$$


Inequalities (B.5) and (B.7) imply $-\left\|V-V_{\gamma}^{*}\right\| \leq V-T_{\gamma} V \leq\left\|V-V_{\gamma}^{*}\right\|$ and thus the second inequality of the theorem. Inequalities (B.6) and (B.8) imply $-\left\|V-V_{\gamma}^{\pi}\right\| \leq$ $V \ldots T_{\gamma}^{\pi} V \leq\left\|_{i} V-V_{\gamma}^{\pi}\right\|$ and therefore the third inequality of the theorem.

Proof of Theorem 15: We prove the desired bound by applying, in turn, the triangle inequality, Assumptions 1,5, and 4, Theorem 13, and finally Assumption 5 a second time:

$$
\begin{aligned}
\left\|V_{\gamma}^{\pi}-V_{\gamma}^{*}\right\| & \leq\left\|V_{\gamma}^{\pi}-T_{\gamma} V\right\|+\left\|T_{\gamma} V \cdots V_{\gamma}^{*}\right\| \\
& =\left\|T_{\gamma}^{r \pi} V_{\gamma}^{\pi}-T_{\gamma}^{\pi} V\right\|+\left\|T_{\gamma} V-T_{\gamma} V_{\gamma}^{*}\right\| \\
& \leq \gamma \cdot\left\|V_{\gamma}^{\pi}-V\right\|+\gamma \cdot\left\|V-V_{\gamma}^{*}\right\| \\
& <\frac{\gamma}{1 \cdots \gamma} \cdot\left\|V-T_{\gamma}^{\pi} V\right\|+\frac{\gamma}{1-\gamma} \cdot\left\|V-T_{\gamma} V\right\| \\
& -\frac{\gamma}{1-\gamma} \cdot\left\|V-T_{\gamma} V\right\|+\frac{\gamma}{1-\gamma} \cdot\left\|V-T_{\gamma} V\right\|-\frac{2 \gamma}{1-\gamma} \cdot d_{B}(V) .
\end{aligned}
$$

To see that this bound cannot be tightened any further, consider a deterministic MDP with two states, 1 and 2 , and two actions, 1 and 2 . Action 1 causes a state transition to state 1 no matter which state it is executed in. Similarly, action 2 always causes a state transition to state 2. All immediate costs are two, except for the execution of action 2 in state 2 , which costs nothing. Now consider the value function $V$ that is defined by $V(1)=$ $V(2)=\frac{1}{1 \gamma}$. From Assumption 7 it follows that $I_{\gamma} V(1)-V(1)-2+\gamma V(1)-V(1)=1$ and $T V(2) \quad V(2)-0+\gamma V(2)-V(2)=-1$. Therefore $d_{B}(V)-i \mid T_{\gamma} V-V \|_{1}=1$. The stationary policy $\pi$ with $\pi(1)=1$ and $\pi(2)-2$ is greedy for $V$ according to Assumptions 5 and 7 . Assumptions 6 and 8 imply $V_{\gamma}^{*}(1)-2, V_{\gamma}^{n}(1)-\frac{2}{1}$, $V_{\gamma}^{*}(2)-0$, and $V_{\gamma}^{\pi}(2)=0$. It follows that $\|\left. V_{\gamma}^{\pi} \quad V_{\gamma}^{*}\right|_{i} \cdots \frac{2 \gamma}{1 \cdots \gamma}=\frac{2 \gamma}{1 \cdots \gamma} \cdot d_{B}(V)$.

Proof of Theorem 16: We prove the desired bound by applying, in turn, the triangle inequality. Assimptions 1. 5. and 4. Theorem 13. Assumption 5 again and finally Theorem 13 a second time:

$$
\begin{aligned}
\left\|V_{\gamma}^{\pi} \quad V_{\gamma}^{*}\right\| & \leq\left\|V_{\gamma}^{\pi}-T_{\gamma}^{\pi} V\right\|+\left\|T_{\gamma}^{\pi} V \cdot V_{\gamma}^{*}\right\| \\
& -\gamma_{i} \mid T_{\gamma}^{\pi}-V_{\gamma}^{\pi} V\|+\| T_{\gamma} V-T_{\gamma} V_{\gamma}^{*} \| \\
& \leq \gamma \cdot \mid V_{\gamma}^{\pi}-V \|+\gamma \cdot d_{M}(V) \\
& \leq \gamma \cdot \frac{\mid V+T_{\gamma}^{\pi} V \|}{1+\gamma}+\gamma \cdot d_{M}(V) \\
& \leq \gamma \cdot \frac{(1+\gamma) \cdot \| V-V_{\gamma}^{*} \mid}{1-\gamma}+\gamma \cdot d_{M}(V) \\
& -\gamma \cdot \frac{(1+\gamma) \cdot d_{M}(V)}{1-\gamma}+\gamma \cdot d_{M}(V)
\end{aligned}
$$




$$
-\frac{2 \gamma}{1-\gamma} \cdot d_{M}(V)
$$

To see that this bound cannot be tightened any further, consider again the Markov decision problem that we used in the proof of Theorem 15, but this time in conjunction with the value function $V$ that is defined by $V(1)=V(2)=1$. Assumptions 6 and 8 imply that $V_{\gamma}^{*}(1)-2$ and $V_{\gamma}^{*}(2)=0$. Since $V_{\gamma}^{*}(1)-V^{\nu}(1)=2-1-1$ and $V_{\gamma}^{*}(2)-V(2)=0-1=-1$, it follows that $d_{M}(V)=\left\|V_{\gamma}^{*}-V\right\|=1$. The stationary policy $\pi$ with $\pi(1)=1$ and $\pi(2)=2$ is greedy for $V$ according to Assumptions 5 and 7. Assumption 8 implies $V_{\gamma}^{n}(1)=\frac{2}{1-\gamma}, V_{\gamma}^{n}(2)=0$, and consequently, $\left\|V_{\gamma}^{n}-V_{\gamma}^{*}\right\|-$. $\frac{2 \gamma}{1-\gamma}-\frac{2 \gamma}{1-\gamma} \cdot d_{M}(V)$

Proof of Theorem 17: From Theorem 13 and Assumption 5 we obtain $\left\|V \cdot V_{\gamma}^{\pi}\right\| \leq$ $\left\|V-T_{\gamma} V\right\| \cdot \frac{1}{1-\gamma}=d_{B}(V) \cdot \frac{1}{1-\gamma}$. Therefore,

$$
V>V_{\gamma}^{\pi} \cdots d_{r}(V) \cdot \frac{1}{1-\gamma} \text {. }
$$

We can now prove the desired bound by applying, in turn, Assumptions 1,3 , and 5, Inequality (B.9), and finally Assumptions 3, 2, and 1.

$$
\begin{aligned}
V_{\gamma}^{*} & =T_{\gamma} V_{\gamma}^{*}>T_{\gamma} V-T_{\gamma}^{\pi} V \\
& \geq T_{\gamma}^{\pi}\left(V_{\gamma}^{\pi}-\frac{d_{B}(V)}{1-\gamma}\right)=I_{\gamma}^{\pi} V_{\gamma}^{\pi}-\frac{\gamma}{1-\gamma} \cdot d_{B}(V) \\
& =V_{\gamma}^{\pi}-\frac{\gamma}{1-\gamma} \cdot d_{B}(V) .
\end{aligned}
$$

Together with Assumption 6 we obtain $0 \leq V_{\gamma}^{*}-V_{\gamma}^{*}<\frac{1}{1-\gamma} \cdot d_{B}(V)$ and, therefore, $\left\|V_{\gamma}^{\pi}-V_{\gamma}^{*}\right\|<\tau_{i-\gamma}^{\gamma} \cdot d_{B}(V)$.

To see that this bound cannot be tightened any further, consider again the MDP that we used in the proof of Theorem 15, but this time in conjunction with the value function $V$ that is defined by $V(1)=V(2)-0$. Assumptions 6 and 8 imply that $V_{r}^{*}(1)-2$ and $V_{\gamma}^{*}(2) \approx 0$ and, therefore, $V_{\gamma}^{*}(1)-2 \geq V(1)$ and $V_{\sim}^{*}(2)-0 \geq V(2)$. From Assumption 7 it follows that

$$
\begin{aligned}
& I_{\gamma} V(1) \quad V(1)=2-0-2 \\
& T_{\gamma} V(2) \quad V(2)-0-0-0 .
\end{aligned}
$$

Therefore $d_{1 s}(V)-\left\|T_{\gamma} V-V\right\|-2$. The stationary policy $\pi$ with $\pi(1) \cdots 1$ and $\pi(2)$ - 2 is greedy for $V$ according to Assumptions 5 and 7 , and Assumption 8 implies $V_{\gamma}^{\pi}(1)-\frac{2}{1-\gamma}$ and $V_{\gamma}^{\pi}(2)=0$. We can conclude that $\| V_{\gamma}^{\pi} \cdots V_{\gamma}^{*} \mid-\frac{2 \gamma}{1-\gamma}=\frac{\gamma}{1-\gamma_{\gamma}} \cdot d_{B}(V)$.

Proof of Corollary 1: The desired bound is an immediate consequence of Theorem 17 in conjunction with the second inequality of Theorem 11. The example used to prove 
Theorem 17 proves that the bound is tight, because it satisfies $d_{B}(V)=d_{M}(V)$.

LFmma 1 Let $V$ be a value function that satisfies $V \leq T_{\gamma} V$. Then $V \leq V_{\gamma}^{*}$.

Proof: Consider mappinge $T_{r, n}$ from $\mathcal{R}^{S}$ to $\mathcal{R}^{S}$ that are inductively defined as follows:

$$
\forall n \in \mathrm{N} \forall V \in \mathcal{R}^{S}: T_{\gamma, 0} V-V, \quad T_{\gamma, n+1} V=T_{\gamma}\left(T_{\gamma, n} V\right) .
$$

It follows by induction from Assumption 3 that $V \leq T_{\gamma, n} V$ for all $n$ if $V \leq T_{\gamma} V$. Similarly, it follows by induction from Assumption 1 that $T_{\gamma: n} V_{\gamma}^{*}=V_{\gamma}^{*}$ and from Assumption 4 that $\left\|T_{\gamma, r} V_{\gamma}^{*}-T_{\gamma, n}^{\gamma} V\right\| \leq \gamma^{n}\left\|V_{\gamma}^{*}-V\right\|$, for all \%. Put together, we can conclude that $\left\|V_{\gamma}^{*}-T_{\gamma, n} V\right\| \leq \gamma^{n}\left\|V_{\gamma}^{*}-V\right\|$ for all $n$, which further implies $\lim _{n \rightarrow \infty} T_{\gamma, n} V=V_{\gamma}^{*}$ and, finally, $V \leq V_{\gamma}^{*}$.

Proof of Corollary 2: The desired bound is an immediate consequence of Lemma 1 and Theorem 17. The example used to prove Theorem 17 proves that the bound is tight, because it satisfies $V \leq T, V$ according to Equations (B.10) and (B.11).

Proof of Corollary 3: The desired bound follows immediately from Lemma 1 and Corollary 1. The example used to prove Theorem 17 proves that the bound is tight, because it satisfics $V \leq T_{\gamma} V$ according to Equations (B.10) and (B.11) and, furthermore, $d_{B}(V)-d_{M}(V)$.

Proof of Theorem 18: The following four inequalities follow from Assumptions 10 and 13 in conjunction with the triangle inequality.

$$
\begin{aligned}
& \left\|Q-Q_{\gamma}^{*}\right\| \leq\left\|Q-B_{\gamma} Q\right\|+\left\|B_{\gamma} Q-Q_{\gamma}^{*}\right\| \leq\left\|Q-B_{\gamma} Q_{\|}\right\|+\gamma \cdot \mid Q \cdots Q_{\gamma}^{*} \| ; \\
& \left\|Q-B_{\gamma} Q\left|\leq\left\|Q-Q_{\gamma}^{*}\right\|+\left\|Q_{\gamma}^{*} \quad B_{\gamma} Q\right\|<\left\|Q-Q_{\gamma}^{*}\right\|+\gamma \cdot\right| Q_{\gamma}^{*} \quad Q\right\|_{i} \\
& \left|i Q \cdot Q_{\gamma}^{\pi}\|\leq\| Q \quad B_{\gamma}^{\pi} Q\|+\| B_{\gamma}^{\pi} Q-Q_{\gamma}^{\pi}\|\leq\| Q-B_{\gamma}^{\pi} Q_{i}\right|+\gamma \cdot\left|Q \quad Q_{\gamma}^{\pi}\right| \mid \\
& \left|Q Q \cdot B_{\gamma}^{\pi} V\right| \leq\left|\left\|Q-Q_{\nu}^{\pi}\right\|-\left\|Q_{\gamma}^{\pi} \quad B_{\gamma}^{\pi} Q\right\| \leq\left\|Q-Q_{\gamma}^{\pi}\right\|+\gamma \cdot \| Q_{\gamma}^{\pi} \cdots Q\right|^{2} .
\end{aligned}
$$

Inequalitics (B.12), (B.13), (B.14), and (B.15) imply the first, second, third, and fourth inequality of this theorem, respectively.

Proof of Theorem 19: The first and third incquality have already been proven in the context of Theorem 18. From Assumptions 12 and 10, we obtain

$$
\begin{aligned}
& B_{\gamma} Q-\left|Q-Q_{\gamma}^{*}\right|: \leq B_{\gamma} Q_{\gamma}^{*}-\left|Q Q-Q_{\gamma}^{*}\right|:=Q_{\gamma}^{*}-|| Q-Q_{\gamma}^{*} \mid<Q ; \\
& B_{\gamma}^{\pi} Q \quad\left|Q \cdots Q_{\gamma}^{\pi}\right| \leq B_{\gamma}^{\pi} Q_{\gamma}^{\pi} \cdot\left|Q-Q_{\gamma}^{\pi}\right|\left|\quad Q_{\gamma}^{\pi} \quad \| Q \quad Q_{\gamma}^{*}\right| \leq Q .
\end{aligned}
$$


Assumptions 10 and 13 imply

$$
\begin{gathered}
\left\|Q_{\gamma}^{*} \quad B_{\gamma} Q\right\|-\left\|B_{\gamma} Q_{\gamma}^{*}-B_{\gamma} Q\right\| \leq \gamma \cdot\left\|Q_{\gamma}^{*}-Q\right\| \leq\left\|Q-Q_{\gamma}^{*}\right\|, \\
\left\|Q_{\gamma}^{\pi}-B_{\gamma}^{\pi} Q\right\|-\left\|B_{\gamma}^{\pi} Q_{\gamma}^{*}-B_{\gamma}^{\pi} Q\right\| \leq \gamma \cdot\left\|Q_{\gamma}^{*}-Q\right\| \leq \| Q \cdot Q_{\gamma}^{*} \mid:
\end{gathered}
$$

and therefore in conjunction with Assumption 15

$$
\begin{array}{rll}
Q \cdots B_{\gamma} Q \leq Q_{\gamma}^{*}-B_{\gamma} Q \leq \| Q_{\gamma}^{*} & \cdots B_{\gamma} Q\|\leq\| Q-Q_{\gamma}^{*} \| ; & \text { (B. 18) } \\
Q-B_{\gamma}^{\pi} Q<Q_{\gamma}^{*} \quad B_{\gamma}^{\pi} Q \leq Q_{\gamma}^{\pi}-B_{\gamma}^{\pi} Q \leq\left\|Q_{\gamma}^{\pi} \quad B_{\gamma}^{\pi} Q\right\| \leq\left\|Q \cdots Q_{\gamma}^{\pi}\right\| & \text { (B. 19) }
\end{array}
$$

Inequalities (B.16) and (B.18) imply - $\left\|Q-Q_{\gamma}^{*}\right\| \leq Q \cdots B_{\gamma} Q<\left\|Q \cdots Q_{\gamma}^{*}\right\|$ and thus the second incqualiiy of the theorem. Inequalities (B. 17) and (B. 19) imply $-\left|Q-Q_{\gamma}^{n}\right| \mid<$ $Q-B_{\gamma}^{\pi} Q<\mid Q \quad Q_{\gamma}^{\pi} \|$ and therelore the third inequality of the theorem.

Proof of Theorem 20: Wc prove the desired bound by applying, in turn, the triangle incquality, Assumption 9, Theorems 3,6 and 18, and finally Assumption 14:

$$
\begin{aligned}
& \left\|V_{\gamma}^{\pi}-V_{\gamma}^{*}\right\| \leq\left|V_{\gamma}^{\pi}-V_{Q}\right|+\mid V_{Q}-V_{\gamma}^{*} \| \\
& -\left\|V_{Q_{\gamma}^{\pi}, \pi}-V_{Q ; r} \mid+\right\| V_{Q}-V_{Q_{\psi}} \| \\
& \leq\left\|Q_{\gamma}^{\pi} \cdot Q\right\|+\left\|Q-Q_{\gamma}^{*}\right\| \\
& <\frac{\left\|Q \cdots B_{\gamma}^{\pi} Q\right\|}{1-\gamma}+\frac{\| Q \quad B_{\gamma} Q !}{1 \cdots \gamma} \\
& -\frac{2 \| Q \quad B_{\gamma} \dot{Q} \mid}{1-\gamma}-\frac{2}{1 \cdots \gamma} \cdot d_{B s}(Q) .
\end{aligned}
$$

To see that this bound cannot be tightened any further, consider a deterministic MDP with a single state 1 and two actions, 1 and 2, that both cause self transitions and have immodiate costs 2 and 0 , respectively. Now consider the $Q$-function $Q$ that is defined by $Q(1,1)=(Q(1,2)=-1 /(1, y)$. From Assumption 16 it follows that $B, Q(1,1)$ $Q(1,1)-2+\cdots\left(V_{Q}(1)-Q(1,1)-2+\frac{\gamma}{1 \gamma}-\frac{1}{1 \gamma}-1\right.$ and $B_{\gamma} Q(1,2) \quad Q(1,2)-$ $0 \cdot \gamma \cdot V_{Q}(1) \quad Q(1,2)=\frac{\gamma}{1-\gamma} \quad \frac{1}{1-\gamma}=1$. Therefore $a_{B}(Q)-\beta_{\gamma} Q-Q \|-1$. The stationary poticy $\pi$ with $\%(1)-1$ is grecdy for $Q$, and Assumplions 6 and 8 imply $V_{*}^{*}(1) \therefore \%(1 \quad \gamma)$ and $V_{\gamma}^{*}(1)-0$. It follows that $\left|V_{\gamma}^{*}-V_{\gamma}^{*}\right| 2 /(1-\gamma)$ $2 d_{B}(Q) /(1-y)$

Proof of Theorem 21: We prove the desired bound by applying, in turn, the triangle incquality, Assumption 9, Theorems 3,6 and 18, Assumption 14, and finally Theorem 18 a second time:

$$
\begin{aligned}
& \left|V_{\gamma}^{\pi}-V_{\gamma}^{*}\right| \leq\left|V_{\gamma}^{*}-V_{Q * *}\right||-| V_{Q} \cdot V_{\gamma}^{*} \mid
\end{aligned}
$$

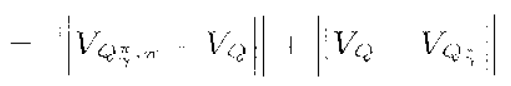




$$
\begin{aligned}
& \leq\left\|Q_{\gamma}^{\pi} \cdot Q\right\|+\left\|Q \cdots Q_{\gamma}^{*}\right\| \\
& \leq \frac{\left\|Q-B_{\gamma}^{*} Q\right\|}{1-\gamma}+\left\|Q-Q_{\gamma}^{*}\right\| \\
& =\frac{\left\|Q \cdot \frac{B}{\gamma} Q\right\|}{\gamma}+d_{M}(Q) \\
& \leq \frac{(1+\gamma) \cdot d_{M}(Q)}{1-\gamma}+d_{M}(Q) \\
& =\frac{2}{1-\gamma} \cdot d_{M}(Q) .
\end{aligned}
$$

To see that this bound cannot be tightened any further, consider again the MDP that we used in the proof of Theorm 20, but this time in conjunction with the $Q$ function $Q$ that is defined by $Q(1,1) \ldots Q(1,2)-1$. The stationary policy $\pi$ defined by $\pi(1) \cdots 1$ is greedy for $Q$ and Assumptions 6 and 8 imply that $V_{\gamma}^{\pi}(1)=2 /(1 \ldots \gamma)$ and $V_{\gamma}^{*}(1)-0$. Assumptions 9,10 , and 16 imply $Q_{\gamma}^{*}(i, a)=c_{i}^{a}+\gamma \cdot V_{Q_{\gamma}^{*}}\left(j_{i}^{a}\right)=c_{i}^{a}+\gamma \cdot V_{\gamma}^{*}\left(j_{i}^{a}\right)$ for all deterministjc MDPs, states $i$, and actions $a \in A(j)$ Hence, $Q_{\gamma}^{*}(1,1) \quad 2$ and $Q_{\gamma}^{*}(1,2)=0$ for the example. It follows that $d_{M}(Q)=\left\|Q_{\gamma}^{*}-Q\right\| \cdots 1$ and, finally, ||$V_{\gamma}^{\pi}-V_{\curlyvee}^{*} \mid=2 /(1-\gamma)-2 d_{M}(Q) /(1-\gamma)$.

Proof of Theorem 22: Let $i$ be an arbitrary state. We prove the desired bound by applying, in turn, Assumptions 6 and 9. Theorems 2, 3, 6, and 18, and tinally Assumption 14.

$$
\begin{aligned}
\left\|V_{\gamma}^{\pi}-V_{\gamma}^{*}\right\| & \leq V_{\gamma}^{\pi}(i) \cdots V_{\gamma}^{*}(i) \\
& =V_{Q_{\gamma}, \pi}(i)-V_{Q_{\gamma}^{*}}(i) \leq V_{Q_{\gamma}, \pi}(i) \cdots V_{Q}(i) \\
& =V_{Q_{*}^{*},}(i)-V_{Q, \pi}(i) \leq \mid V_{Q_{\gamma}^{m}, \pi}-V_{Q, \pi} \| \\
& \leq \mid Q_{\gamma}^{\gamma_{i}}-Q \| \leq \frac{\left\|Q-B_{\gamma}^{\pi} Q\right\|}{1 \cdots \gamma} \\
& -\frac{\mid Q-B_{\gamma} Q \|}{1}-\frac{d_{\beta}(Q)}{1-\gamma}
\end{aligned}
$$

To sec that this bound cannot be tightened any further, consider again the MDP that we used in the proof of Theorems 20 and 21 , but this time in conjunction with the Q-function $Q$ that is definct by $Q(1,1)-Q(1,2)=0$. In the proof of Theorem 21 , we showed that $V_{\gamma}^{*}(1)-0, Q_{\gamma}^{*}(1,1)=2$, and $Q_{\gamma}^{*}(1,0)=0$. Therefore, $Q<Q_{\gamma}^{*}$. Assumption 16 implies that $B, Q(1,1)-Q(1,1)=2+\gamma \cdot V_{Q}(1)-Q(1,1) \cdots z_{z}$ $D_{\gamma} Q(1,2)-Q(1,2)=0 \cdots, V_{Q}(1)-Q(1,2)-0$, and therefore $\left.d_{t} ; Q\right) \ldots i D_{\gamma} Q \quad Q !$ 2. The stationary policy $\pi$ with $\pi(1)=1$ is greedy for $Q$. Assumptions 6 and 8 imply $V_{\gamma}^{*}(1)-2 /(1-\gamma)$, and, therefore, $\left\|V_{\gamma}^{\pi}-V_{\gamma}^{*}\right\|-2 /(1-\gamma)-d_{B}(Q) /(1-\gamma)$. 
Proof of Corollary 4: The desired bound is an immediate consequence of Theorems 22 and 19. The example used to prove Theorem 22 proves that the bound is tight, because it satisfics $Q_{\gamma}^{*}(1,1)-2, Q_{\gamma}^{*}(1,2)-0$, and therefore $a_{B}(Q)=a_{M}(Q)$.

LeMma 2 Let $Q$ be a $Q$-function that satisfies $Q<B_{\gamma} Q$. Then $Q<Q_{\gamma}^{*}$

Proof: Consider mappings $B_{\gamma, n}$ from $\mathcal{R}^{M}$ to $\mathcal{R}^{M}$ that are inductively defined as follows:

$$
\forall n \in \mathrm{N} \forall Q \in \mathcal{R}^{M}: B_{\gamma, 0} Q=Q, \quad B_{\gamma, n+1} Q-B_{\gamma}\left(B_{\gamma, n} Q\right) .
$$

It follows by induction from Assumption 12 that $Q \leqslant B_{\gamma, n} Q$ for all $n$ if $Q<B_{\gamma} Q$. Simikarly, it follows by induction from Assumption 10 that $B_{\gamma, r} Q_{\gamma}^{v} \cdots Q_{\gamma}$ and from Assumption 13 that $\left\|B_{\gamma, n} Q_{\gamma}^{*}-B_{\gamma, n} Q\right\| \leq \gamma^{n}\left\|Q_{\gamma}^{*} \quad Q\right\|$, for all $n$. Put together, we can conclude that $\left\|Q_{\gamma}^{*}-B_{\gamma, n} Q\right\| \leq \gamma^{n}|| Q_{\gamma}^{*}-Q \|$ for all $n$ which further implies $\lim _{\eta, \cdots \infty} B_{\gamma, n} Q-Q_{\gamma}^{*}$ and, finally, $Q<Q_{\gamma}^{*}$.

Proof of corollary 5: Lemma 2 implies $Q \leq Q_{\gamma}^{*}$. Thus, Theorem 22 can be applied and gives the desired result.

Proof of Corollary 6: Temma 2 implies $Q \leq Q_{\gamma}^{*}$. Thus, Corollary 4 can be applied and gives the desired result.

Proof of Theorem 23: Let $Q$ be an arbitrary Q-function. We will show that for every statc $i$ there exists a real valuc $\epsilon(i)>0$ with the following property: It $\max _{a \in A(i)}\left|Q(i, a) \cdot Q_{\gamma}^{*}(i, a)\right|<\epsilon(i)$ then every action that is greedy for $Q$ and $i$ is also gredy for $Q_{\gamma}^{*}$ and $i$. If such values $\epsilon(i)$ exist, then $c=\min _{i \epsilon, S} \epsilon$ (i) gives the desired result

Let $i$ be any state. If every action in $A(i)$ is greedy for $Q^{*}$ and $i$, then $\epsilon(i)$ can be set to an arbitrary non-zero value, for example one. Otherwise, sct

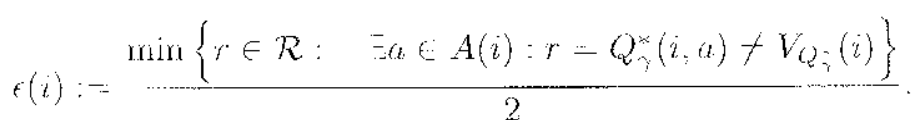

The desired result follows using $\Lambda$ ssumption 17.

Proof of Corollary 7: The coroilary follows immediately from Theorems 23 and 18.

Proof of Theorem 24: From Assumption 17 we conclude that the Q function $Q+2 t$ has the desired property. 
Proof of Corollary 8: The corollary follows immediately from Theorems 24 and 18 .

\section{Notes}

1. In the dynamic programming literature, it is ofter assumed that the immediate cost of a state transition diperrits only on the stauting state and the executed action, but not on the successor state reached. This; is the ascumption that we make in this section. We could casily extend the theory to the more general case, but are afraid that the resulting dynamic programming operators might look umfamiliar to the reader

2. This assumprion is made for convenience. It follows already from Assumptions 1 and 7.

\section{References}

Batird, L.. (1495). "Residual Algorithms: Reinforcement Learning with Function Approximation." Machire Learning. Procedings of the I2th International Conference, Morgan Kaufmann Fublishers. Inc. San FranAisco, CA.

Barto, A. G., Sutton, R. S., \& Anderson, C. W., (1983). "Neuronlike adaptive elements that can solve difficult Garning control problems." It.EE Transactions on Systerts, Man. and Cyberneitss 15: pp. 834-846

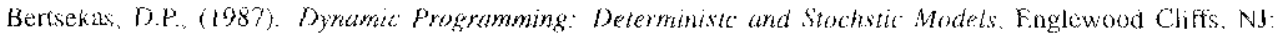
Prentitis: Hali.

Boyau, J. \& Moore, A. (1995) "Generalization in Reinforcement Leaming: Salcly Approximating the Valuc Function:" G. Fesauro, D. S. Toureizky and T. K. Lean editors, Advances in Neurat Informalon Procescurs, Stisterns 7. MIT Press, Carnbridge MA.

Crites, R H. \& Barto, A. G., (1995, unpublished). "Improving Elevator Perfontance Using Reintorceriten Learnirge."

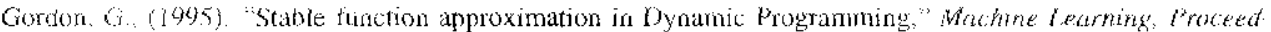

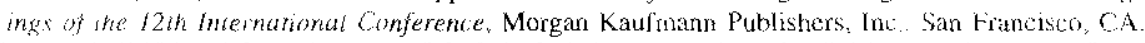

Heger. M (1994a), "Consideraion of Risk in Reinforcement Jeaming:" Machint Learning, Precedings of the The Intemationat Conference pp. 105-111, Morgan Kasfmann Publishers Inc. San Francisco. CA.

Heger. M. (1994b), "Risk and Reinforcement Learning; Concepes and Dynamic Programming" Tecthnicat

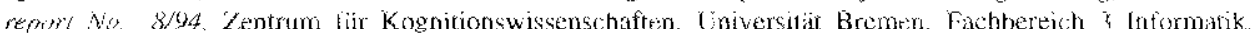
Germiay

Hegcr, M. (1995), "Risk and Ranforcemenr Learning: Algonithns," Technical repon (forthcomming). Zentrum tür Kogrniricuswissenschatten. Universität Bremen. Fachbereich 3 Informatik Cerciany.

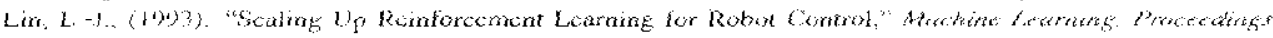
fif the towh Thternetionat Conference pp. 182 189, Morgan Kaufmarm Publeshers Inc. San Francisco. CA.

l.itman. M. L.. (1994). "Markov ganes as a framework for mutri-ageot reintorcement learning." Machine

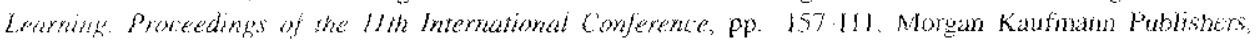
Inc. San fiancisco, C.A

Mcronald, M. A. F. \& Hingeon P., (1994). "Approximate Discounied Dynariti: Programming Is Vhreliablo;" Teckmial report 04/6. Linversity of Western Austalid.

Mont. A.W. \& Atkeson C. G. (to appear). "The Parti game Alyorithm for Vhriable kesolution Reintorcenti leameng in Mulidimensional State spaces" Machine learning

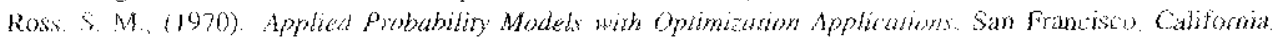
Holder bay.

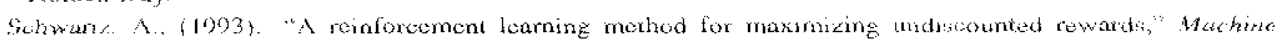

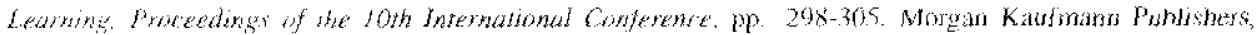
Inc. San Francisco, CA

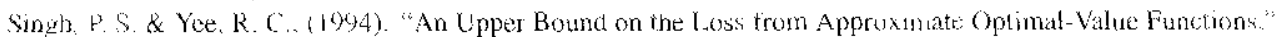

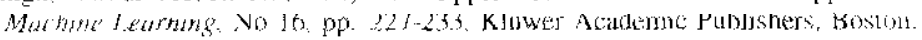


Sutton, R. S., (unpublished). "Generalization in Reinforcement Learning: Successful Examples C'sing Sparse Coarse Coding."

Taha, H. A., (1987). Opoerations Research: An Introduction, Fourth Fitition. Macmillan Publishing Compary New York

Tesauro, G. J. (1992). "Pracical Issues in Temporal Difference Learning," Machine Learring. 8(3/4). pp 257.277 .

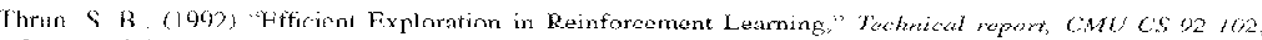
School of Cormpuier Science, Carnegie Mellon Liniversity, Pittsburgh, PA.

Thrun, S. B. \& Schwart\%, A., (1993). "Issues in Using Function Approximation for Reinforcement Learning:" Proceedings of the Farth Connectionist Models Summer Schoot, Lawrence Erlbaum Publishcrs, Hillsdale. NJ

Tsitsiklis, J. N., (1994). "Asynchronous Stochastic Approximation and Q-I carning," Machine learning. No 16. pp. 185-202, Kluwer Academic Publishers, Boston.

Watkins, C. J C. H. (1989). Leaming from Delayed Rewards, Phi) thesis, Cambridge Universiry Fngland watkins, L. J. C. H \& Uayan, P., (1992). "Q-Learning," Machine Learning, No 8, pp. 279.292.

Williams, R.I. \& Baird III, I.C., (1993). "Tight Performance Bounds on Greedy Policis Based on Impertect Value Functions," Technical Report NU.CCS-93-14, Northeastern University.

Zhang, W. \& Dietterich, T. G., (1995). "A Reinforcement Learning Approach to Job-shop Scheduling." Pro. ceedings of the Ith International Joint Conference on Artificial Intelligence. Morgan Kaufmann Publishers, Inc., San Francisco, CA.

Received November 8.1994

Accepted February 24, 1995

Final Manuscripe October 6, 1995 